\title{
SUR UN POINT DE VUE HEURISTIQUE CONCERNANT LA PRODUCTION ET LA TRANSFORMATION DE SUPPORTS AU PALÉOLITHIQUE MOYEN
}

\author{
Ludovic SLIMAK*
}

\begin{abstract}
Mots-clés. Paléolithique moyen, technologie, typologie, méthodologie, Levallois, Discoïde, Kostienki, Néronien.
Résumé. Cet article aborde les principes épistémologiques propres à l'analyse des principaux systèmes techniques du Paléolithique moyen. La réflexion porte sur les notions de prédétermination Levallois, de récurrence, de rythme ou encore de formules prédéterminantes. Leurs attributions sont exposées et discutées. Le fruit de cette réflexion est l'élaboration d'un outil méthodologique visant à s'extraire du principe dualiste - Levallois versus non Levallois - propre aux analyses des systèmes techniques du Paléolithique moyen. L'application de cet outil est illustrée au travers de la présentation de concepts originaux. Du point de vue des outillages, sont discutées les notions de catégories, de fonctionnalité et de rationalité des produits finis. Cette analyse vise à approcher certains principes de l'outil moustérien.
\end{abstract}

Key-words. Middle Palaeolithic, technology, typology, methodology, Levallois, Discoid, Kostienki, Neronian.

Abstract. This paper addresses some key principles in Mousterian lithic technology with a particular focus on the notions of Levallois predetermination, recurrence, rhythm and "formules prédéterminantes" (predetermining formulae). The acceptance of these notions in the profession is presented and discussed. This reflection has led to the development of a methodological tool distinct from the dualist principle of analysis - Levallois versus non Levallois - specific to studies of Mousterian technical systems. The application of this tool is illustrated through a presentation of original flaking concepts. Concerning typological analysis, the notions of categories, functionality and the rationality of end-products are discussed. The objective of this analysis is to compare some aspects of Mousterian tools with Upper Palaeolithic behaviours.

Translation: Magen O'FARRELL

Schlüsselwörter. Mittelpaläolithikum, Technologie, Typologie, Methodologie, Levallois, Diskoidal, Kostienki, Néronien. Zusammenfassung. In diesem Artikel werden die für die Analyse der wichtigsten technischen Systeme des Mittelpaläolithikums charakteristischen erkenntnistheoretischen Prinzipien behandelt. In der Reflexion geht es um die Begriffe prédétermination Levallois, Rekkurenz, Rhythmus oder formules prédéterminantes, deren Eigenschaften und Anwendungen dargestellt und diskutiert werden. Das Ergebnis dieser Reflexion ist die Erstellung einer Methodologie, die versucht sich aus dem dualistischen, den Analysen der technischen Systeme des Mittelpaläolithikums eigenen Prinzips - Levallois versus nicht Levallois - zu lösen. Die Anwendung dieser Methode wird anhand bislang noch nicht identifizierter Reduktionskonzepte dargestellt. Hinsichtlich der Werkzeuge werden die Kategorien, die Funktionalität und die Rationalität der Endprodukte diskutiert. Diese Analyse versucht gewisse Prinzipien des Moustérien-Werkzeugs zu verstehen.

Übersetzung: Isa ODENHARDT-DONVEZ

\footnotetext{
* UMR 5608 du CNRS : «TRACES », Maison de la recherche, Université Toulouse-II-Le Mirail, 5 allées Antonio-Machado, F-31058 Toulouse Cedex 9. Courriel : slimak@univ-tlse2.fr
} 
Les procédés d'obtention de supports reconnus pour le Paléolithique moyen sont particulièrement peu nombreux. Les objectifs de production s'expriment autour de trois grandes catégories, éclats, lames et pointes qui sont largement assujettis à une classification binaire: Levallois et non Levallois. Ces débitages rendent compte d'une grande part des systèmes d'exploitation des roches siliceuses reconnus pour cette période. Dans le même temps, il faut relever l'existence d'angles d'approche globalement antagonistes quant à leur acceptation. Différents courants peuvent ainsi être différenciés selon que l'on confère à ces systèmes un champ d'application plus ou moins large.

Ce travail ne propose ni une classification ni un dénombrement des procédés d'obtention de supports reconnus pour le Paléolithique moyen. Le très riche débat autour de la variabilité et de l'extension des systèmes Levallois et Discoïdes n'est pas au cour de cette synthèse qui a pour vocation d'exposer des réflexions non conclusives concernant les procédés d'obtention de supports au Paléolithique moyen et leur insertion dans un cycle de confection d'outillage.

\section{PRODUCTION DE SUPPORTS AU PALÉOLITHIQUE MOYEN}

\section{GÉOMÉTRIE DU NUCLÉUS ET NOTION DE PRÉDÉTERMINATION}

Depuis les travaux d'Éric Boëda, les débitages Levallois sont perçus comme une vaste entité technique dont l'unité repose sur une identité conceptuelle (Boëda, 1993 et 1994). Le succès de la méthode s'est principalement exprimé autour de l'appropriation de quelques idées princeps de cette réinterprétation du concept. C'est en l'occurrence la structure et la géométrie du nucléus qui ont connu le plus grand succès dans les analyses ultérieures des ensembles à composante Levallois. Si dans cette méthode analytique, les produits finis (les supports Levallois de plein débitage) gardent une valeur diagnostique, dans son application la notion de prédétermination du support est reléguée à un plan secondaire. Le produit fini n'est plus au cour de la définition du système. Ce basculement des centres d'intérêt des préhistoriens, de l'objectif artisanal vers le nucléus a plusieurs origines.

L'une de ces origines est probablement historiographique, résultant d'une certaine désaffection quant à l'analyse des produits finis, liée au premier développement des analyses technologiques au milieu des années 1980. De manière connexe, on relèvera que les attributs des produits finis sont qualitatifs, donc discutables. Aucun éclat n'est intrinsèquement Levallois. La Levalloisité exprime donc un état relatif. En tant que tel, il représente une propriété inférée. En l'absence de remontage, l'attribution de cette propriété repose sur un diagnostic qualitatif illustrant une projection de l'analyste sur les objectifs de production supposés du Préhistorique. À l'inverse, les attributs de caractérisation du nucléus Levallois s'appuient sur des principes géométriques simples et définis en tant qu'invariants. Sur cette base une architecture Levallois des nucléus est définie et appréhendée comme l'élément princeps du concept.

La confrontation de ces deux approches explique en partie l'attention portée aux nucléus et un certain désintérêt vis-à-vis des produits finis et des objectifs de débitage. On peut alors opposer l'évaluation du caractère Levallois d'un produit - essentiellement lié à sa morphologie et sa régularité -, aux critères discriminants de reconnaissance du nucléus Levallois - quelques points de géométrie élémentaire distinguent le nucléus Levallois des autres nucléus. Ce réagencement des méthodes de l'analyse exprime alors un passage du qualitatif au quantitatif.

C'est à partir de la géométrie des nucléus que la question d'un recoupement du concept Levallois avec d'autres systèmes de débitage s'est finalement structurée, au travers de la comparaison des propriétés des débitages Levallois et Discoïdes. Ces réflexions peuvent être résumées en une question très simple: l'axe du plan de détachement d'un support sur un nucléus induit-il le caractère Levallois d'un débitage? Dans le détail, les nucléus présentant une corniche périphérique, une hiérarchisation stricte entre surface de débitage et surface de réserve, ainsi qu'une table de débitage plane sont-ils nécessairement des nucléus Levallois? La question pourrait paraître anodine si elle ne remettait en cause l'un des fondements de cette approche, à savoir le caractère invariant et exclusif de cette construction du nucléus.

Ces interrogations se sont exclusivement portées ces dernières années sur les liens de causalité existant entre débitages Discoïdes et Levallois récurrents centripètes. De fait, les nucléus discoïdes, tels qu'ils étaient entendus par François Bordes correspondent, en grande partie, aux nucléus Levallois récurrents centripètes décrits par Éric Boëda. Il s'agit du débitage moustérien de François Bordes, pour lequel le nucléus est "préparé comme s’il s'agissait d'un nucléus Levallois » et s'en distinguant essentiellement dans le déroulement du débitage: « au lieu d'enlever un grand éclat après préparation [...], on continue à enlever des éclats centripètes"(Bordes, 1961). 
Ces nucléus discoïdes ont donc les mêmes caractères qu'un nucléus Levallois, présentant une hiérarchisation stricte entre surface de débitage et de réserve. La géométrie de la surface de débitage est elle-même similaire à celle d'un nucléus Levallois (fig. 1) amenant l'auteur à envisager des termes de passage d'un mode de débitage vers l'autre.

Dans cette discussion autour de la valeur de l'architecture du nucléus, on relèvera par ailleurs que, quelles que soient les démarches adoptées, les analyses d'ensembles archéologiques sont largement inductives. Les approches se revendiquent, a priori, d'un courant de pensée par un exercice de renvois bibliographiques sans évaluer, au cas par cas, ce qui ressort intrinsèquement de débitages Levallois, Discoïdes ou autres. La réflexion s'articule alors autour de la reconnaissance d'une variabilité comportementale de thèmes très généraux. Les fondements conceptuels des démarches analytiques employées sont peu discutés, jamais évalués.

Il en ressort que la notion de prédétermination, notion princeps des débitages Levallois, a perdu une grande partie de sa substance, faute d'être l'objet d'évaluations contradictoires systématiques. Si tout support ne peut être considéré comme intrinsèquement prédéterminé, qu'entend-on exactement par prédétermination Levallois?
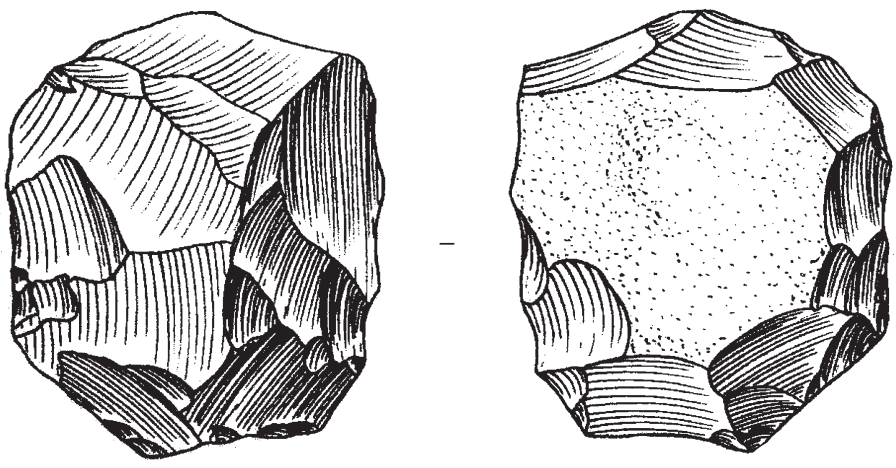

1
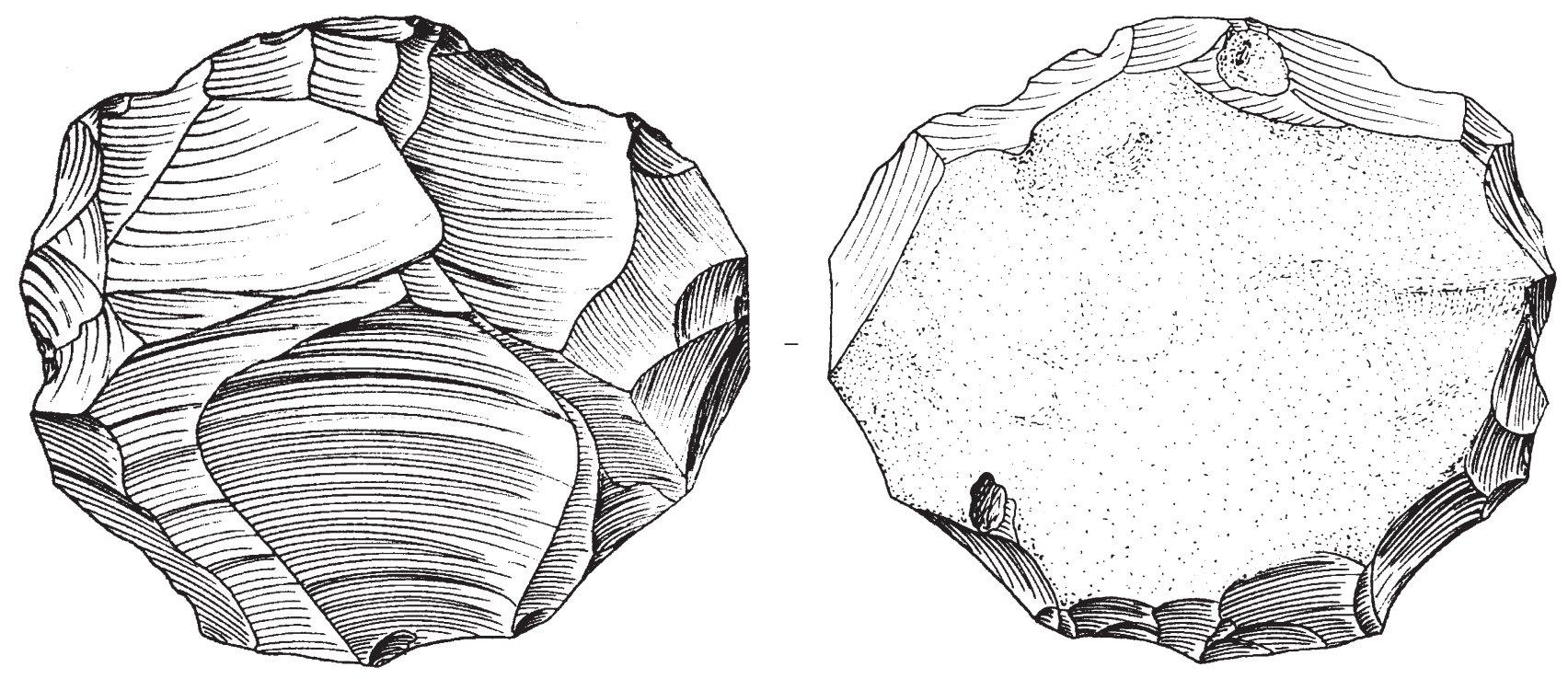

2

0 $5 \mathrm{~cm}$

Fig. 1 - Nucléus discoïdes présentant tous les caractères de ce qui sera, par la suite, défini en tant que nucléus Levallois récurrents centripètes (d'après F. Bordes, 1961). 


\section{RYTHMES ET RÉCURRENCE DU CONCEPT LEVALLOIS}

Paradoxalement, si le produit Levallois est devenu le parent pauvre des démarches analytiques, il reste le tenant de la philosophie du concept. L'unité du système s'enracine profondément dans l'idée du ou des support(s) préférentiel(s). Ce principe s'exprime dans une notion technique d'ordonnance du débitage en vue de l'obtention de supports dont les propriétés géométriques sont spécifiquement établies. Cette idée de produit dont l'ensemble des caractères morphologiques exprime la volonté de l'artisan est particulièrement évidente dans le cadre d'un débitage ayant pour finalité l'obtention d'un unique support, qu'il s'agisse d'un éclat, d'une lame ou d'une pointe. À la suite de l'enlèvement du support préférentiel (Levallois), les propriétés géométriques du volume exploitable sont à restaurer. La caractérisation de la notion de récurrence par É. Boëda permet d'aborder la diversité de ces systèmes de production sous l'angle conceptuel. Le concept Levallois se décline alors, au fur et à mesure du développement de nos connaissances des sociétés du Paléolithique moyen, en une très riche famille de méthodes. L'unité du système, divers sur tous les autres plans, repose sur la notion de prédétermination au sein de nucléus présentant un plan équatorial.

Il y a, sur cette seule notion, plusieurs possibilités d'ambiguité.

Tout support résultant d'un débitage anthropique est, de par sa nature, prédéterminé. Il ne faudrait donc pas parler de support prédéterminé, terme inadapté, mais de la notion de prédétermination Levallois, ce qui est singulièrement différent. Cette notion sous-entend que la volonté de l'artisan s'exprime, dans le déroulement opératoire, par la succession de phases d'aménagement et de plein débitage. La notion de récurrence Levallois se limite alors à l'extraction de supports prédéterminés entre deux phases chronologiques de gestion des caractères géométriques du volume exploité.

La notion que nous pourrions appeler récurrence absolue est antinomique avec le principe de récurrence Levallois. Il faut entendre par récurrence absolue l'idée selon laquelle il existerait des débitages Levallois au sein desquels chaque support est à la fois prédéterminant et prédéterminé. Expérimentalement, il existe effectivement des principes de débitage simples permettant l'obtention de supports globalement normalisés sans faire appel à une notion de prédétermination Levallois. Ce mode d'exploitation d'un bloc repose sur la répétition d'actions élémentaires, sans notion de prédétermination. Ces débitages ne ressortent pas d'un même principe dynamique. Prenons un exemple caricatural. Un éclat Kombewa ou une entame corticale ne sont pas des supports Levallois. Certes, mais pourquoi?

Car ces supports, prédéterminés (!), ne résultent pas d'une notion de prédétermination Levallois. Il faut entendre par là que ces éclats sont extraits volontairement (!) et que leur morphologie générale est connue et maîtrisée par l'artisan préalablement à leur extraction. Ils sont donc investis d'une notion de prédétermination. Ces supports sont le résultat d'une production raisonnée et maîtrisée induite par un volume existant mais qui ne résulte pas d'une phase technique préalable ayant vocation à exprimer les propriétés futures du support. Les propriétés morphologiques du produit sont conjoncturelles. Techniquement, au sein des débitages Levallois, il n'existe pas de support investi d'une prédétermination spécifique sans la possibilité d'établir une hiérarchie objective entre des phases de mise en place d'une convexité et des phases de plein débitage Levallois.

Les débitages Discoïdes peuvent être inversement appréhendés comme résultant d'une logique de consommation des convexités d'une table de débitage. Ce principe met en lumière l'absence d'une réelle stratégie dans l'agencement des différents enlèvements lors du déroulement opératoire.

Corollairement, les débitages Levallois sont marqués par une anticipation réelle - entendre objective - dans l'ordonnancement des enlèvements. Cette anticipation est clairement absente au sein du concept Discoïde, ou bien se trouve limitée à sa plus simple expression: la gestion progressive des enlèvements sur la zone exploitée.

Ce parallèle illustre une même règle de base: il y a une dichotomie fondamentale, quantifiable et incontournable entre les notions de consommation et de gestion raisonnée et anticipée d'un matériau ${ }^{1}$.

Les débitages au sein desquels la notion de prédétermination est inexistante (ou induite) ne sont pas des débitages Levallois. S'il en était autrement, la notion même de débitage Levallois, en tant que concept, n'aurait plus lieu d'exister. Cela n'exclut pas, dans l'application de ces principes de base, l'existence de raccourcis techniques dans la mise en place et la gestion du caractère de prédétermina-

1. Cette notion de consommation d'une convexité revient à Jacques Pelegrin, suite à une discussion tenue à Bağlama (Cappadoce) durant l'été 2002. 
tion, mais ces procédés doivent alors être évalués et discutés au cas par cas, en fonction des réalités et des contextes rencontrés. La démonstration de la présence d'un débitage Levallois au sein d'une série archéologique ne peut reposer sur la reconnaissance de quelques principes géométriques au sein des nucléus et un jeu de renvois bibliographiques. Les réalités archéologiques sont riches et donc peu sujettes à s'ordonner de manière binaire.

Ce principe d'alternance dans le déroulement du débitage constitue un des fondements du concept et ressort, d'ailleurs, de l'ensemble des études proposées par É. Boëda. Cette idée peut être illustrée par la découverte au milieu des années 1980 des débitages laminaires dans les séries de Rocourt et Seclin. Ces débitages ont été attribués dans un premier temps à une expression du Levallois (Boëda, 1988), puis individualisés du concept Levallois par le même auteur car chaque enlèvement crée et entretient les conditions techniques nécessaires à la poursuite du débitage, jusqu'à exhaustion, sans passer par des phases de restauration des convexités de la table de débitage. La distinction de ces débitages vis-à-vis du concept Levallois était inéluctable afin « que la définition du débitage Levallois reste opérationnelle et discriminante » (Boëda, 1990).

Ce qui est valable pour les débitages laminaires l'est tout autant pour les productions d'éclats. L'idée, selon laquelle certains débitages Levallois seraient caractérisés par l'obtention de supports à la fois prédéterminants et prédéterminés, marque un glissement sémantique dans l'appréhension de ces systèmes. À ma connaissance, cette notion n'a jamais été employée par É. Boëda que dans le cadre de débitages, certes récurrents, mais limités à l'obtention de quelques supports car « très vite, après trois ou quatre bons enlèvements, il est nécessaire de repréparer la surface de débitage si on veut poursuivre le débitage et refaire une deuxième série d'enlèvements » (Boëda, 1990). Cette déviation du sens original accordée à ces débitages génère des contresens quant au champ d'application des débitages Levallois et ses limites en tant que concept.

Force est de constater que la notion de débitages Levallois à récurrence absolue repose sur un élargissement du concept. Cette extension épistémologique est ambiguë, car implicite; aucune analyse n'aborde le déroulement d'un tel processus opératoire. Ces approches renvoient cependant systématiquement à la définition établie par É. Boëda en 1993, bien que cette dernière soit clairement structurée à partir d'un principe technique antinomique: «à une phase de production de supports prédéterminés Levallois succède une phase de réaménagement des convexités » (Boëda, 1993, p. 400).

Ce glissement sémantique trouve probablement son origine dans la place qui a été attribuée aux nucléus pour caractériser les débitages Levallois, et en particulier au rôle accordé au plan de détachement des supports par rapport à la corniche d'un nucléus.

\section{RYTHMES ET RÉCURRENGE DU DÉBITAGE DISCOÏDE EN TANT QUE CONCEPT}

À l'inverse, cette notion de récurrence absolue constitue un des critères structurels des débitages Discoïdes. Chaque support est investi d'un rôle technique d'auto-entretien des surfaces exploitées. Cette notion permet d'élaborer une distinction forte au sein des nucléus à plan équatorial et, en l'occurrence, entre débitages Discoïdes et Levallois.

Ces concepts résultent de choix indépendants et sont caractérisés par des rythmes qui leur sont spécifiques: d'un côté les débitages Discoïdes s'inscrivent dans un rythme continu du débitage, c'est-à-dire au sein duquel il est impossible de hiérarchiser phases d'aménagement des convexités et phases de production de supports prédéterminés; et de l'autre, les productions Levallois répondent à un rythme discontinu où alternent phases de plein débitage et phases de restauration des convexités.

Cette notion de rythme a pour corollaire la notion de prédétermination des supports recherchés. Nous pouvons par ce biais établir une hiérarchisation intrinsèque entre ces débitages. Si le principe régissant les concepts Discoïdes et Levallois repose sur une dynamique spécifique et non sur une catégorisation des nucléus, l'équation Nucléus $\Leftrightarrow$ Concept, telle qu'elle a été largement employée, n’a plus lieu d'être.

Dès lors, il n'y a plus aucune raison de limiter cette notion exclusivement aux débitages centripètes. Certains débitages unipolaires ou bipolaires ressortent non seulement d'une même dynamique, mais s'expriment sur des nucléus présentant une même architecture. Cette proposition est d'autant plus pertinente qu'il existe au sein des débitages Discoïdes, des modalités de passage entre débitages centripètes et unipolaires, par le biais de l'extraction d'un éclat à crête correspondant à la mise en place d'un plan de frappe préférentiel (Slimak, 2003).

Ces débitages centripètes, unipolaires et bipolaires, résultent d'une même logique technique (fig. 2 et 3 ). Si l'on suit cette proposition, les débitages Discoïdes peuvent être pleinement abordés dans leur valeur conceptuelle. Le plan 

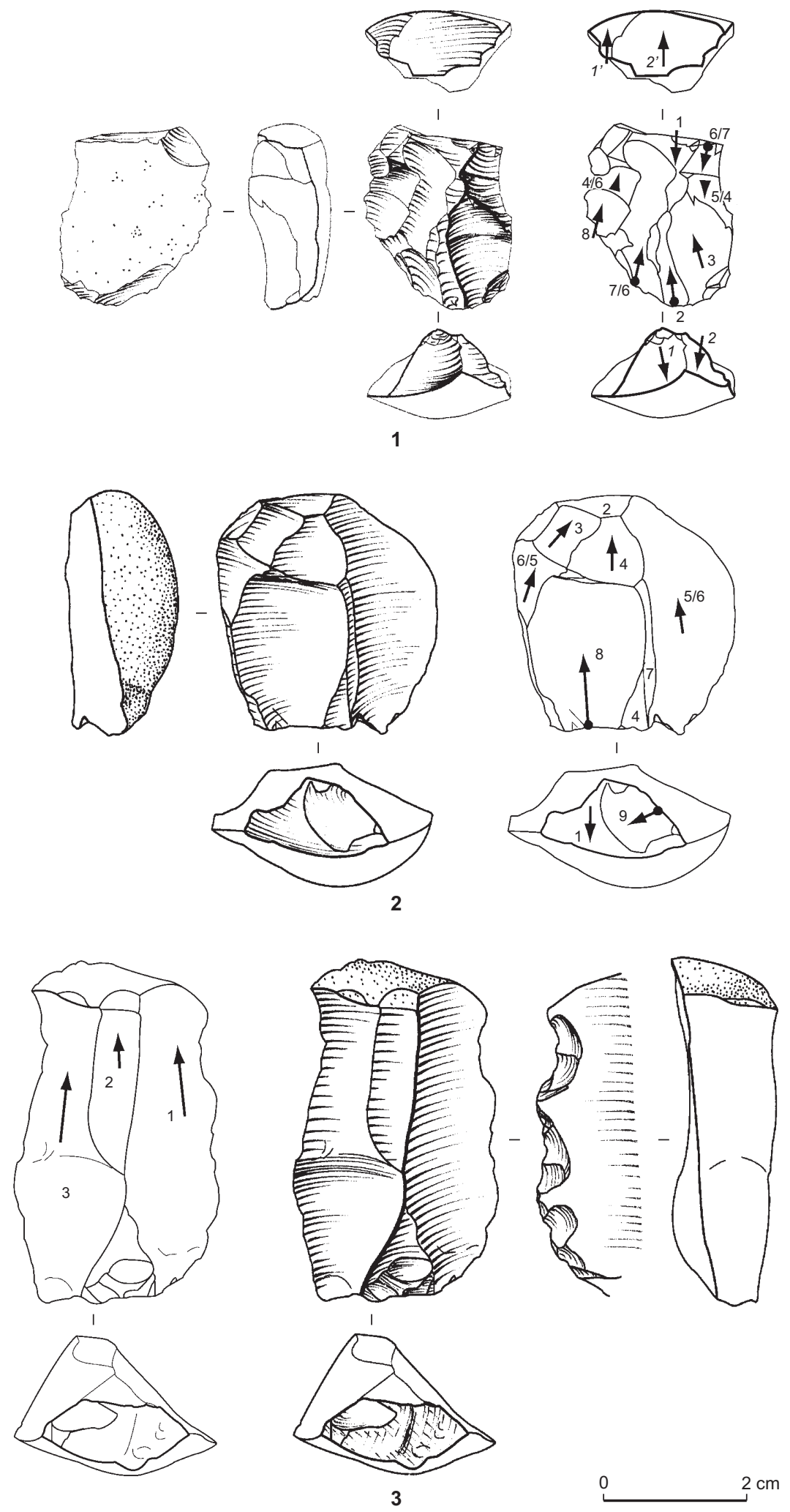

Fig. 2 - Concept Discoïde, méthodes unipolaires et bipolaires : nucléus et produits caractéristiques de Champ Grand à Saint-Jean-Saint-Maurice-sur-Loire (Loire) (dessin : L. Slimak, CNRS). 

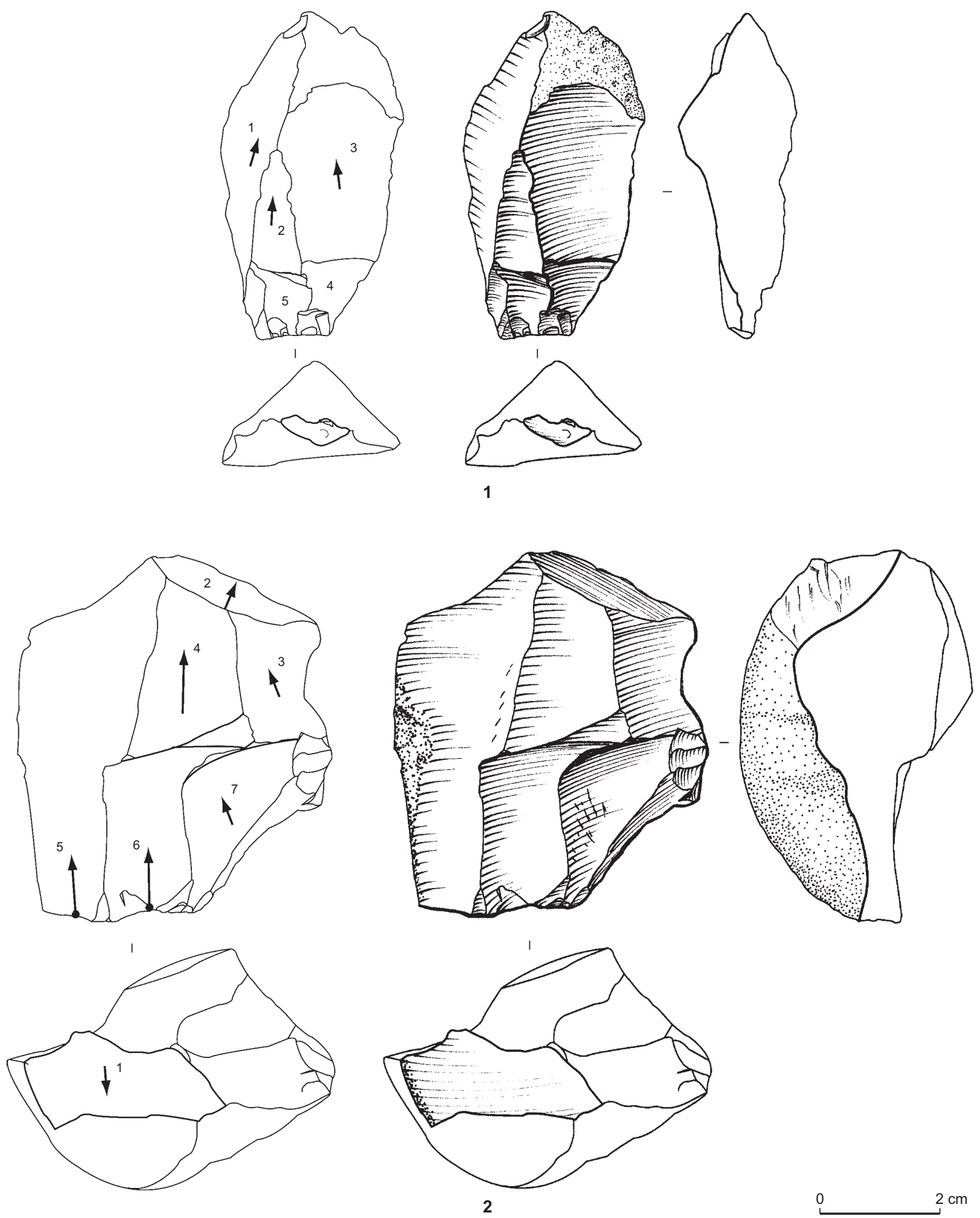

Fig. 3 - Concept Discoïde, méthodes unipolaires et bipolaires : nucléus et produits caractéristiques de Champ Grand à Saint-Jean-Saint-Maurice-sur-Loire (Loire) (dessin : L. Slimak, CNRS). 
de détachement des supports vis-à-vis du plan équatorial des nucléus et la hiérarchisation surface de débitage/plan de frappe ne constituent alors que des modalités dans le déroulement d'un débitage.

D’une manière générale, l'analyse des débitages uni/ bipolaires, à rythme continu, met en évidence des traits redondants: la mise en forme des blocs est systématiquement sommaire, limitée à la mise en place d'un ou deux plans de frappe. La surface exploitée ne nécessite aucun aménagement particulier. Conceptuellement, ces nucléus se distinguent des productions Levallois par cette absence de phase de mise en place des convexités ou de restauration des surfaces exploitées. Ces particularités ont une incidence directe sur la dynamique du débitage tout autant que sur la structure géométrique des nucléus (Slimak, 2003).

Selon cette acception, peut être considéré comme Levallois un débitage mettant en jeu un rythme discontinu, à partir de nucléus dont l'équilibre s'articule autour des principes définis par É. Boëda. Ces critères peuvent alors être appréhendés comme un minimum incompressible quant à la construction géométrique du nucléus mais cette architecture ne constitue plus un facteur exclusif du concept Levallois.

\section{UN EXEMPLE D'APPLICATION: KOSTIENKI ET DÉBITAGES LEVALLOIS}

Le débitage Kostienki, tel qu'il est présenté ici a été reconnu à partir des industries du gisement moustérien de Champ Grand à Saint-Jean-Saint-Maurice-sur-Loire (Loire) . Pour plus de détails, je renvoie le lecteur aux analyses de cet ensemble archéologique (Slimak, 2004; Slimak dir., 2008). Une étude des débitages lamellaires Kostienki est par ailleurs disponible (Slimak, Lucas, 2005).

La principale embûche quant à la reconnaissance de ces éléments est induite par l'existence de deux réalités techniques : débitage et façonnage. Différents critères permettent, dans ce contexte, de distinguer outil et nucléus (Slimak, 2004; Slimak, Lucas, 2005). La démarche consiste à appréhender la structure propre à l'objet par la détermination de sa logique interne. Celle-ci est perceptible grâce à l'analyse de la dynamique générale des enlèvements affectant le support/nucléus.

Nous allons nous intéresser plus particulièrement à distinguer deux grandes familles composant les débitages Kostienki.

\section{STRUCTURE DES NUCLÉUS KOSTIENKI ET NOTION DE PRÉDÉTERMINATION}

Au sein de ces débitages, la morphologie des supports recherchés est dépendante des formules d'agencement établies par l'artisan. Deux modes de débitage peuvent être clairement distingués: des débitages Kostienki à rythme continu et des débitages Kostienki à rythme discontinu.

Les débitages Kostienki à rythme continu génèrent des supports peu standardisés dont la configuration est dépendante du volume original du nucléus, du nombre de plans de frappe, de leur fonctionnement et de l'agencement des enlèvements sur la surface d'exploitation (fig. 4). Ces débitages sont caractérisés par une importante variabilité dans l'agencement des enlèvements, ces agencements n'étant ni systématisés ni modélisables.

On peut opposer à ces débitages des nucléus Kostienki fortement structurés dans leur dynamique et répondant à un rythme discontinu obéissant à de véritables formules prédéterminantes. Ces productions illustrent différents degrés dans la construction des nucléus. Quatre grands moments peuvent être distingués :

1) positionnement de crêtes postéro-latérales conférant au nucléus sa géométrie;

2) aménagement d'un plan de frappe par troncature;

3) extraction de supports débordants et convergents qui mettent en place à la fois les convexités de la zone d'exploitation et dégagent une arête centrale triangulaire;

4) extraction d'une ou deux lamelles pointues et rectilignes qui sont particulièrement investies et peuvent être considérées comme un plein débitage ou, tout au moins, en tant que produits d'intention première (fig. 5).

La géométrie des nucléus et l'agencement des enlèvements se focalisent sur l'obtention de supports fortement prédéterminés: des lamelles pointues ou des micropointes très allongées.

\section{KOSTIENKI OU LEVALLOIS ? PROPRIÉTÉS D'UN CONCEPT KOSTIENKI}

On peut évidemment se demander s'il existe une réelle différence entre les microdébitages Kostienki et les débitages Levallois. En ce qui concerne les productions de lamelles pointues, les formules prédéterminantes mises en œuvre par les artisans confèrent aux produits un net caractère de prédétermination. On relèvera ainsi que ces productions illustrent un attribut particulièrement levalloisien: les blocs les plus investis dans leur structuration 

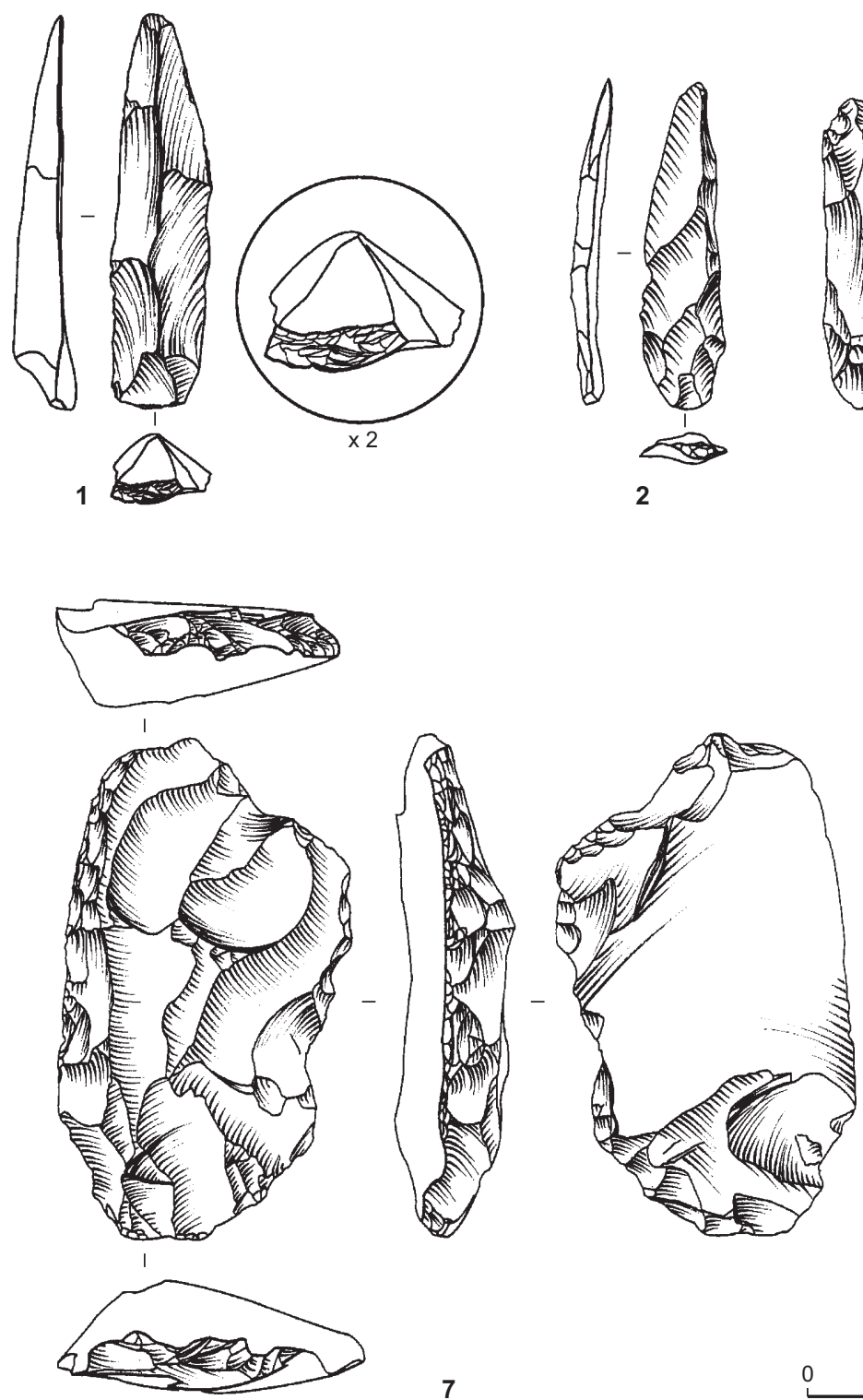

7

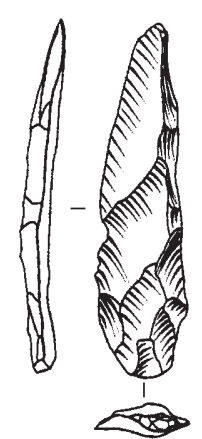

2

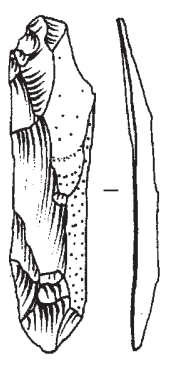

3

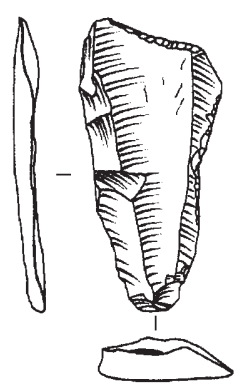

4
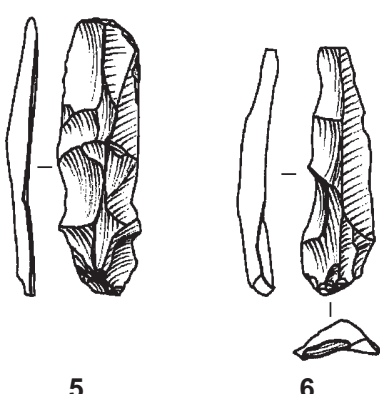

5

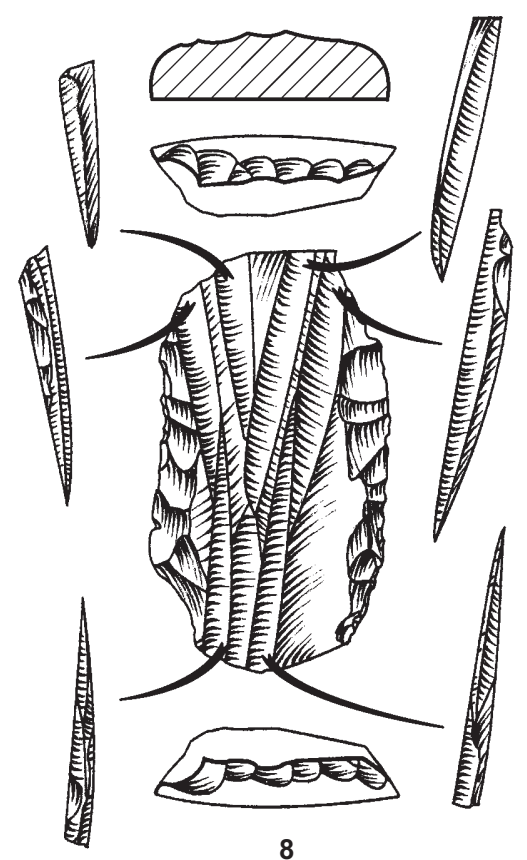

Fig. 4 - Concept Kostienki : production de lamelles de Champ Grand à Saint-Jean-Saint-Maurice-sur-Loire (Loire) (dessin : L. Slimak, CNRS).

sont souvent ceux qui sont le moins intensément exploités. Ce rapport de l'artisan à la matière illustre une spécificité comportementale qui, pour le moins, ne relève pas de l'anecdote.

Dans le même temps, la mise en place des crêtes et la disposition de la surface exploitée en face supérieure d'un éclat leur confère une section transversale fortement asymétrique. Cette configuration ne s'inscrit pas dans la géométrie d'un nucléus Levallois. Les nucléus ne présentent pas un plan équatorial individualisant une surface d'exploitation d'une surface de réserve, mais un unique volume plano-convexe au sein duquel les crêtes ont essentiellement un rôle de délimitation de la zone exploitée. Il n'est alors pas possible de distinguer une surface de débitage d'une surface de réserve. Les deux sont étroitement imbriquées. Ainsi traités, les deux bords latéraux permettent une structuration géométrique du bloc et peuvent fonctionner comme de véritables crêtes postéro-latérales.

Les nucléus Kostienki ne résultent pas de la projection d'un débitage Levallois sur un éclat, mais s'appuient sur une architecture et une dynamique d'exploitation dont l'équilibre ne ressort pas du même principe. 

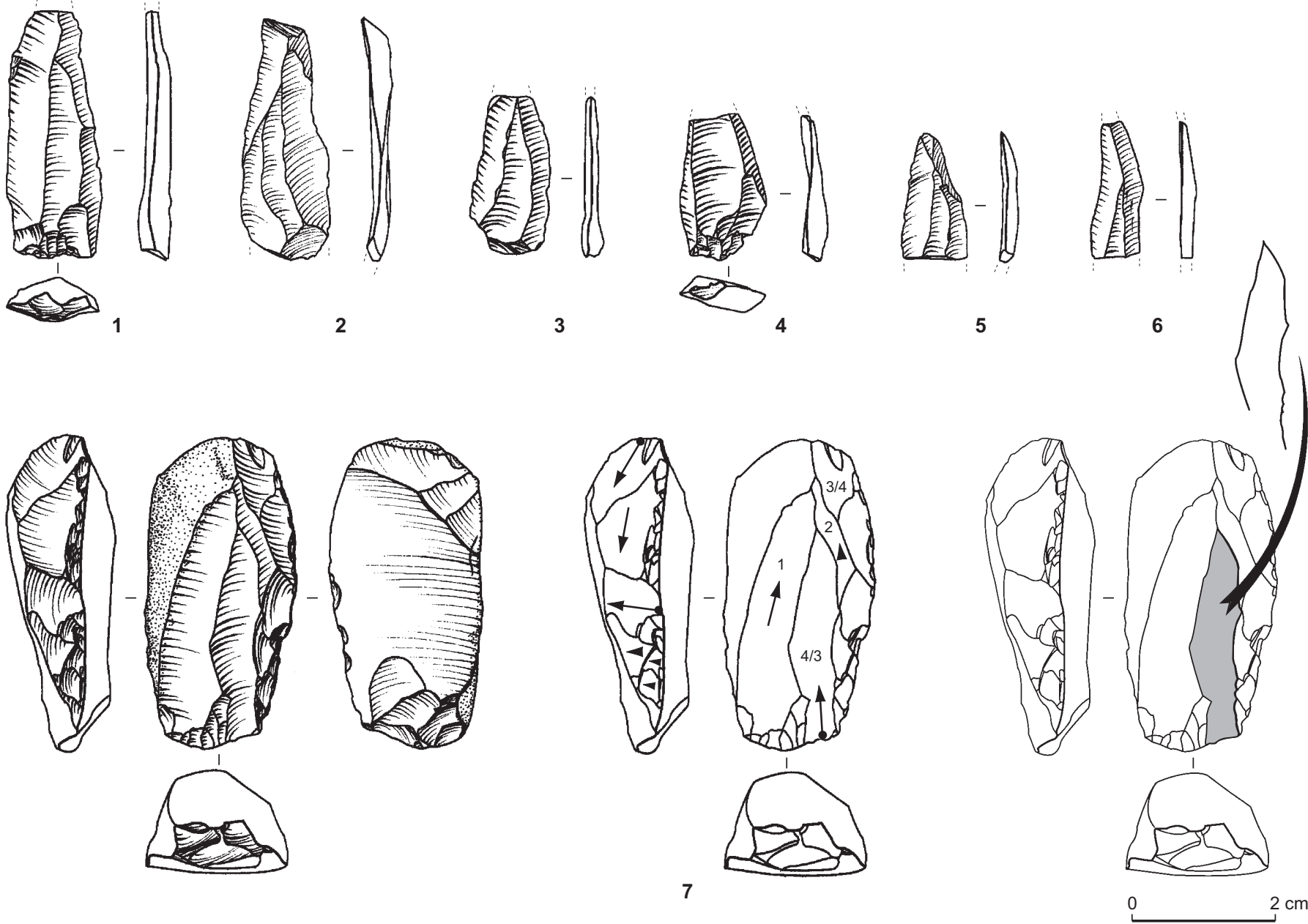

Fig. 5 - Concept Kostienki : production de micropointes très élancées (lamelles pointues) et fortement prédéterminées de Champ Grand à Saint-Jean-Saint-Maurice-sur-Loire (Loire) (dessin : L. Slimak, CNRS).

Le dénominateur commun entre Levallois et Kostienki est donc bien à rechercher dans le rapport de l'artisan à la notion de prédétermination. Ces liens s'expriment dans l'élaboration de formules prédéterminantes équivalentes. De fait, ces formules ne sont pas l'apanage des seuls débitages Levallois, à moins de considérer la notion de prédétermination comme exclusive à ce concept.

Si l'on peut distinguer les débitages Kostienki et Levallois en deux concepts distincts, leur analyse révèle une certaine communauté dans la perception du produit fini par les artisans. La proposition de dénommer ces lamelles des micropointes - très - allongées permet de souligner cette communauté de perception.

Enfin, si les débitages Kostienki sont actuellement reconnus sur éclats et marquent une volonté d'obtenir des supports de très petite dimension, ce système n'est aucune- ment assujetti à ce caractère microlithique. On relèvera, à titre d'exemple, l'existence de débitages Kostienki permettant l'obtention de supports de grande dimension (fig. 6).

Sur un autre plan, l'éclat-support - sur lequel s'établit généralement un débitage Kostienki - ne peut pas être considéré comme un élément discriminant du concept, un tel débitage pouvant s'exprimer, dans son principe, à partir des supports les plus variés. Il est ainsi possible de reconnaître cette exploitation particulière des roches sur des matériaux divers: galets, rognons, plaquettes, etc. La section transversale plano-convexe, résultant d'une absence de surface de réserve - telle qu'on l'entendrait au sein d'un débitage Levallois -, étant alors obtenue soit par sélection de certains blocs pour leur morphologie naturelle, soit mise en place par une phase préliminaire de configuration du bloc (Slimak, 2004; Slimak dir., 2008). 

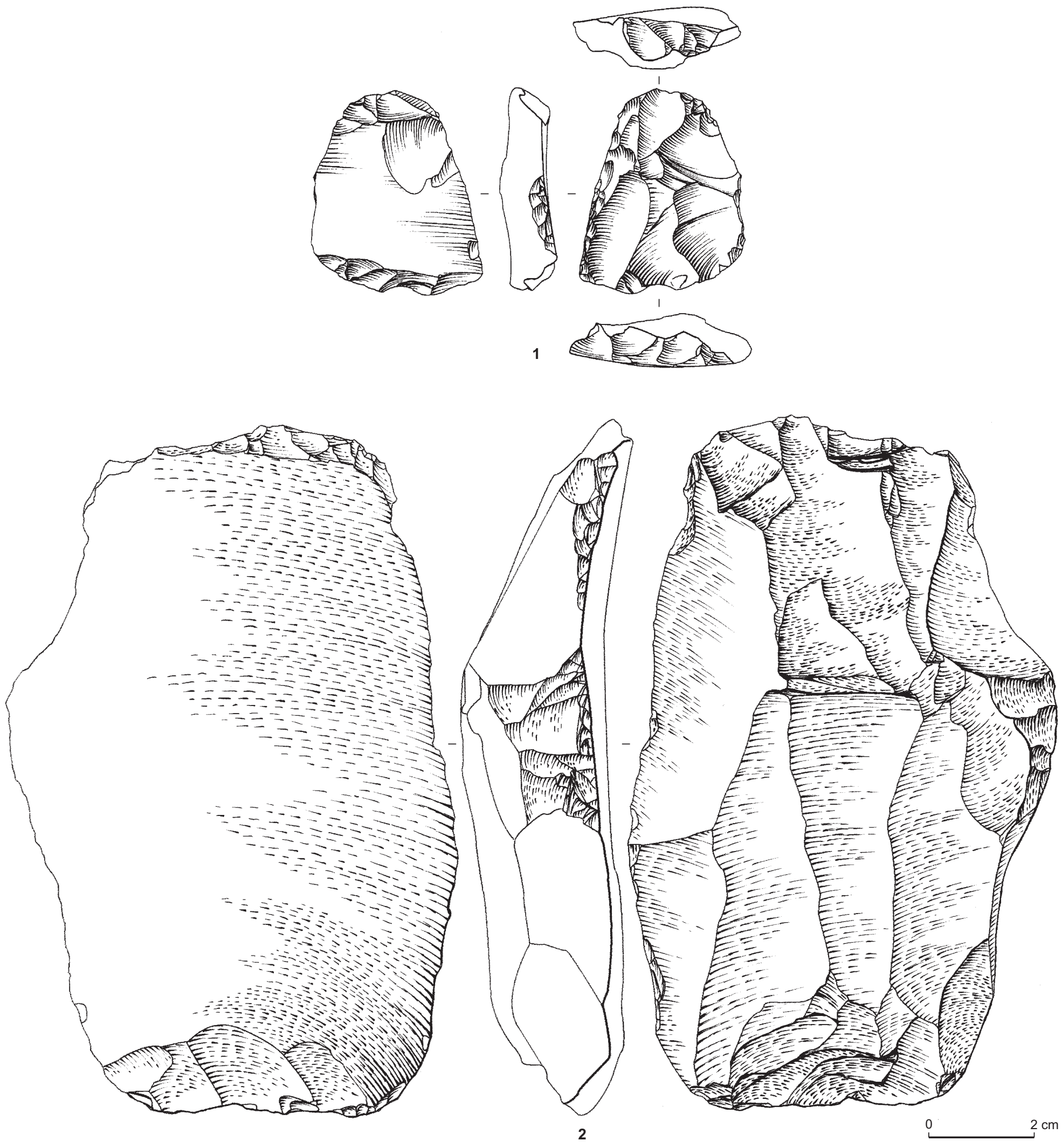

Fig. 6 - Concept Kostienki, nucléus Kostienki : production de supports lamellaires et laminaires; les débitages Kostienki en tant que concept ne présentent aucun lien direct avec leur dimension : 1, Champ Grand à Saint-Jean-Saint-Maurice-sur-Loire (Loire) ; 2, Verrières-les-Buisson (Essonne) (dessin : L. Slimak, CNRS). 


\section{POINTES, POINTES LEVALLOIS, POINTES LEVALLOISIENNES}

\section{SUR LES NOTIONS DE CHOIX ET D'INTENTIONS DES ARTISANS}

En marge du concept Levallois, différents procédés d'obtention de pointes aiguës peuvent être reconnus. Nous allons aborder certains débitages de pointes obtenues par le biais de schémas s'éloignant du concept Levallois classique. Ces systèmes techniques s'expriment dans les ensembles du Néronien ${ }^{2}$, en France méditerranéenne (Slimak, 2004 et 2007).

L'obtention de ces pointes nécessite un fort investissement technique lors de la mise en forme du nucléus. Quel que soit le schéma employé, une phase initiale a pour objectif l'élaboration d'une convergence distale et la mise en place d'un cintrage (convexité transversale) croissant: la partie distale de la surface de débitage doit présenter un dièdre plus marqué que la partie proximale. Cette architecture du nucléus, nécessaire à l'obtention d'une pointe à forte prédétermination, nécessite une mise en forme préalable importante.

Lobjet pointe constitue une finalité en soi; cet objectif peut se décliner selon une dimension variable (fig. $7, \mathrm{n}^{\text {os }} 1$ à 4). Pour autant, le caractère de prédétermination de ces produits est particulièrement marqué et ces débitages ne mettent en jeu qu'une faible notion de récurrence. Le plein débitage repose alors sur l'obtention d'une unique pointe, séparée d'une deuxième phase productive par une restauration du volume du nucléus (a minima table de débitage et plan de frappe). Au premier abord, l'obtention d'une pointe entraîne alors une importante consommation de matière première, par une forte proportion de déchets et/ ou sous-produits. Cette proposition doit immédiatement être réévaluée. Les différents schémas d'obtention de pointes mettent en jeu une notion de deuxième intention - ici des lames (fig. 7 et fig. 8). Cette notion ne doit pas être confondue avec la notion de deuxième choix établie par Jacques Pelegrin concernant certains débitages laminaires (Pelegrin, 1986 et 1995). La notion de deuxième intention repose sur une hiérarchie des produits obtenus, sur la

2. Le Néronien est un groupe culturel défini durant mes recherches doctorales. Il s'agit d'une industrie de « transition » de la fin du Paléolithique moyen propre à la France méditerranéenne, aire où n'est pas représenté le Castelperronien. Le Néronien exprime un des moments de la structure historique complexe, et encore mal connue, de la fin du Moustérien en Europe occidentale. coexistence de différents objectifs, parfois au sein d'une même phase opératoire et sur une gradation de leur prédétermination. Cette dynamique de production s'inscrit mal dans une division binaire distinguant, abruptement, produits et sous-produits.

\section{ORDRE LEVALLOISIEN DE CES TECHNOLOGIES}

Dans ce contexte l'appellation «pointe Levallois » est discutable. Pourquoi?

Le principe de prédétermination mis en évidence dans ces schémas de production est concordant avec un débitage Levallois: il repose sur une alternance entre supports prédéterminants et prédéterminés, et illustre une discontinuité dans le rythme du débitage, critère fondamental d'une prédétermination Levallois.

En revanche, l'architecture générale du nucléus et la gestion de son volume ne s'inscrivent pas dans la variabilité classique d'un nucléus Levallois. Ces nucléus, qui ne s'organisent pas à partir d'un plan équatorial, montrent un développement de la zone d'exploitation qui peut être subpériphérique. Leur amorce peut s'établir à partir d'un dièdre naturel, parfois précédé de l'aménagement d'une crête à double versant préparé. Cette amorce laminaire est suivie d'une structuration du support par une véritable phase laminaire, globalement semi-tournante à partir de blocs de morphologie pyramidale.

Plus qu'une simple modalité de structuration des supports, cette phase laminaire relève d'une véritable volonté artisanale. Ces débitages illustrent donc une démarche en deux temps permettant l'obtention de lames et lames pointues préalablement à l'extraction d'une unique pointe fortement normée.

D'un point de vue structurel, cette dynamique n'est pas contingentée par une architecture Levallois des nucléus. Cette remarque ne doit évidemment pas faire perdre de vue que la vision de la prédétermination, telle qu'elle est ici établie par les artisans, relève, quant à elle, d'un même principe. Il est possible sur ce point d'établir un rapprochement avec les débitages Kostienki qui illustrent l'emploi de formules prédéterminantes d'ordre Levallois sur des nucléus dont l'architecture n'est pas assujettie à la même géométrie.

Ces distinctions ne peuvent être considérées ni comme anecdotiques, ni comme l'expression de modalités au sein d'un même concept de débitage. Elles se font l'écho de particularités techniques fondamentales.

Cette approche volontairement restrictive des débitages Levallois ne doit pas masquer les liens existant quant aux 

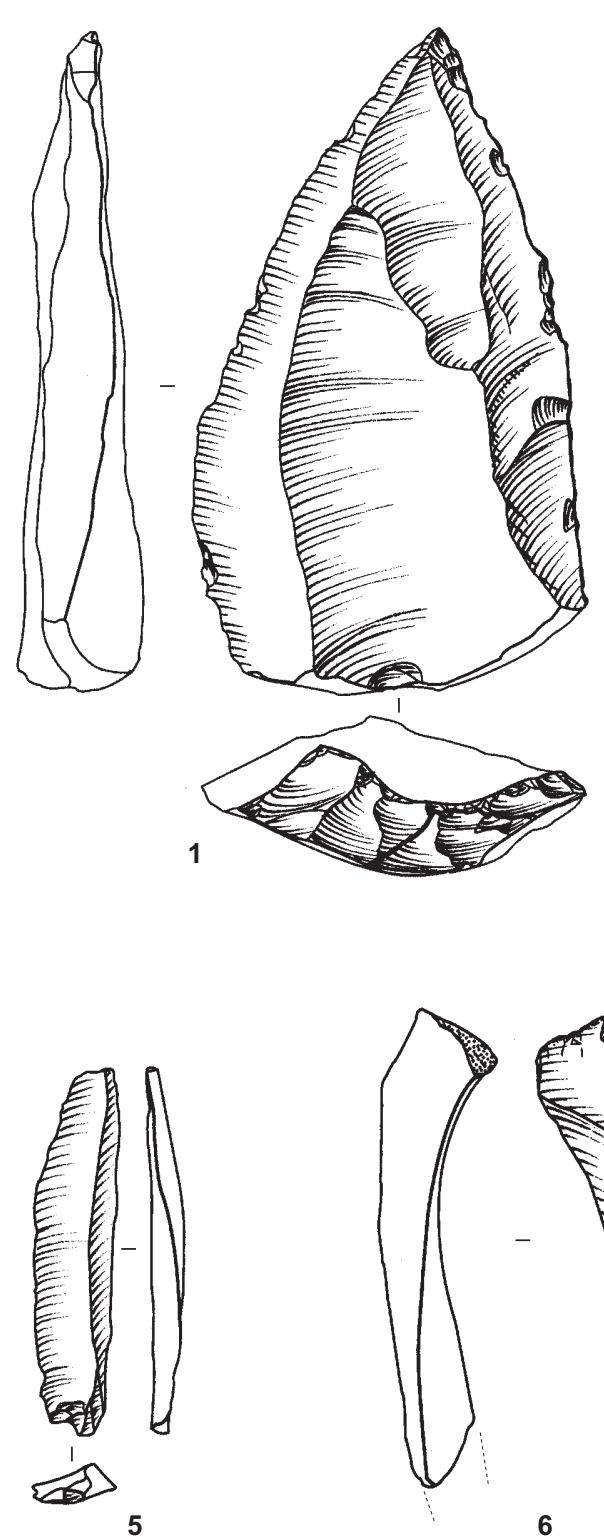

0 $2 \mathrm{~cm}$
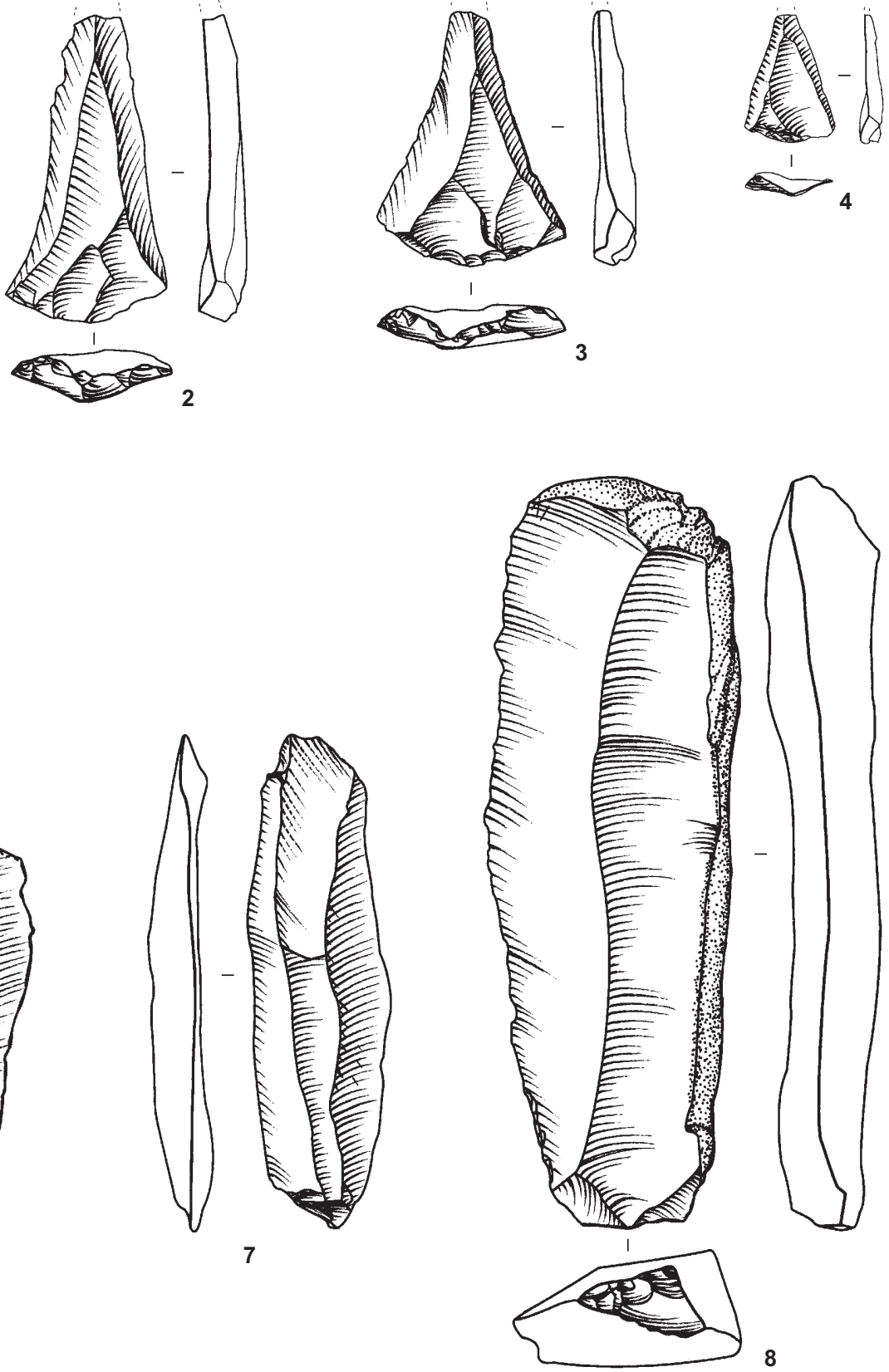

Fig. 7 - Production de pointes «levalloisiennes» du Néronien de la grotte Mandrin à Malataverne (Drôme), niveau 6 (dessin : L. Slimak, CNRS).

modalités établies par les artisans en vue d'atteindre une prédétermination. Il existe ici une véritable cohérence dans le principe même de cette représentation. Pour autant, les particularités techniques de ces débitages induisent une réserve quant à leur rattachement au concept Levallois, ou alors tout débitage mettant en ouvre une notion de prédétermination devrait être considéré comme Levallois. Nous nous trouverions non plus face à un concept de débitage,
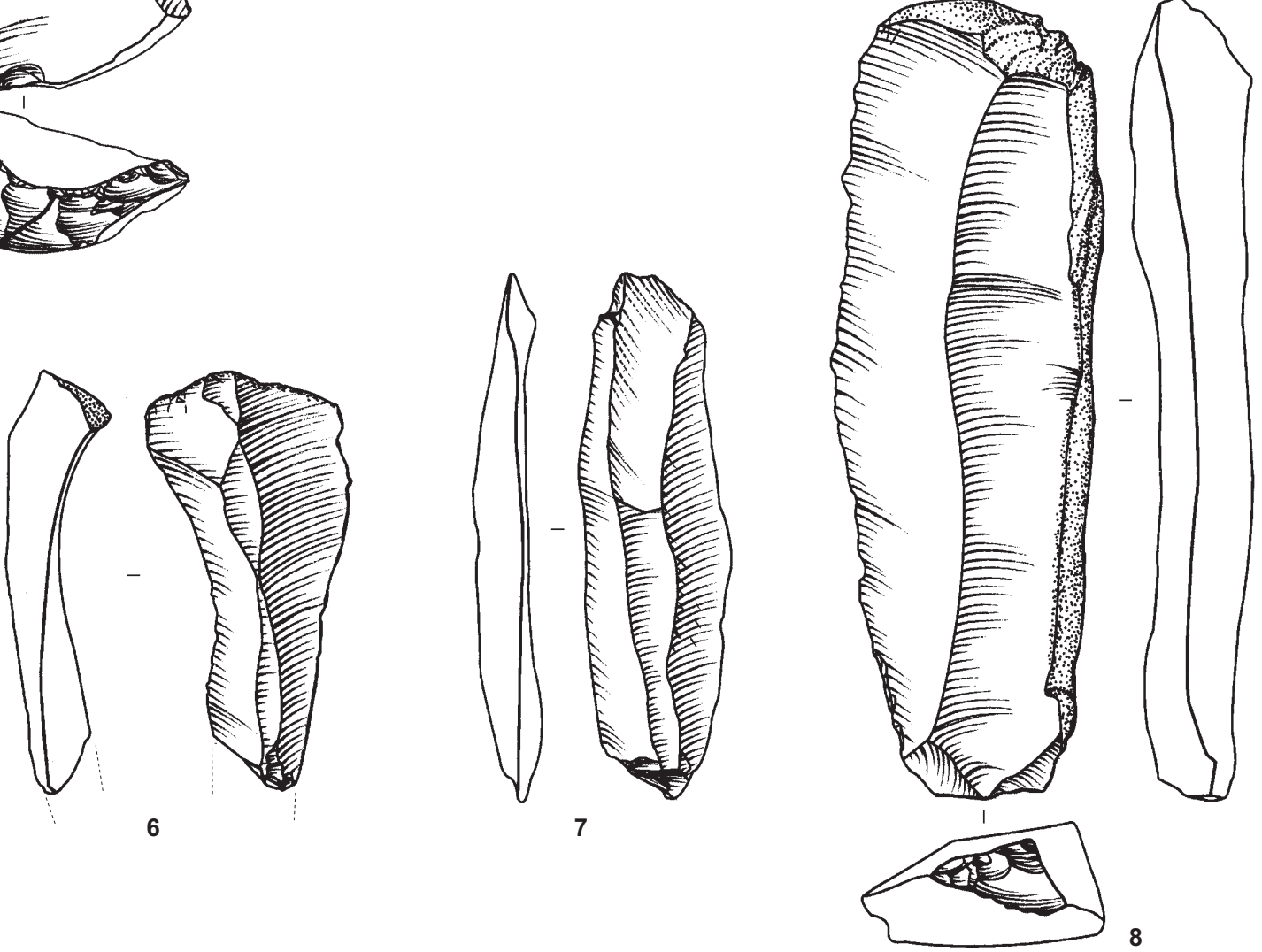

mais face à un métasystème qui, de par sa nature, perdrait de sa valeur diagnostique. Il faut entendre par là qu'un tel système serait autosuffisant; autonome, sinon en opposition, vis-à-vis des réalités archéologiques empiriques.

Le problème soulevé permet donc d'aborder à nouveau, mais sous un autre jour, notre perception des concepts techniques au Paléolithique moyen. Les deux visages des schémas de pointe qui viennent d'être décrits peuvent se 

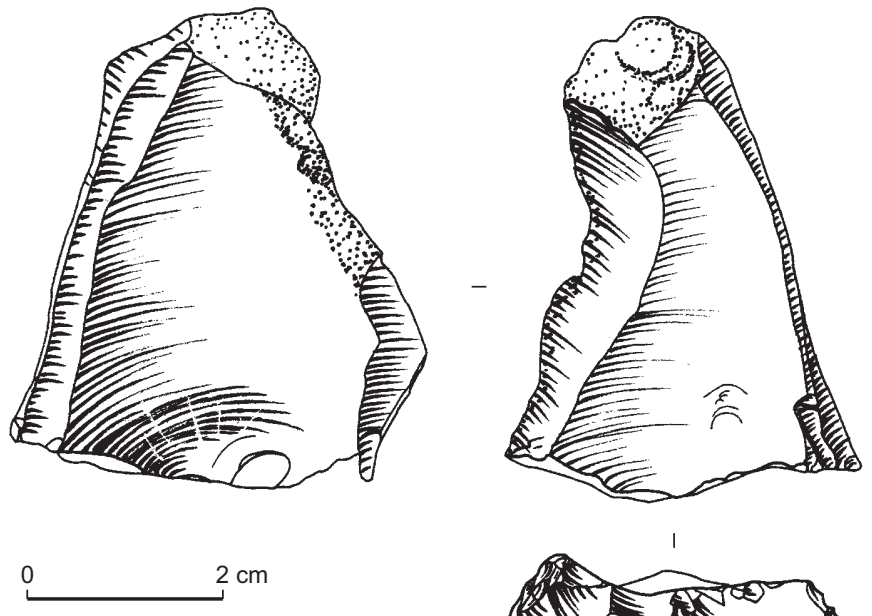

Fig. 8 - Production de pointe "levalloisienne" du Néronien de la grotte Mandrin à Malataverne (Drôme), niveau 6 (dessin: L. Slimak, CNRS).

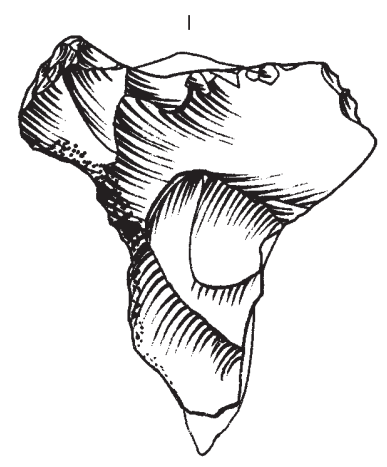

résumer ainsi : l'architecture des nucléus n'est pas Levallois, pas plus que la dynamique de configuration des blocs. La notion de prédétermination est, quant à elle, enracinée dans la même communauté de conception du produit fini que celle s'exprimant au sein d'un débitage Levallois.

Il serait possible de rendre compte du caractère particulier de ces productions par l'emploi de la dénomination de pointe levalloisienne ${ }^{3}$, appellation générique qui soulignerait le décalage technique qui vient d'être abordé.

\section{DE L'OUTIL MOUSTÉRIEN}

\section{L'OUTIL, L'OBJET ET LA CATÉGORIE}

En tant que produit fini, l'analyse typologique de l'outil devrait permettre d'accéder à la notion d'intention de l'artisan. Cependant, la perception de l'outil au Paléolithique moyen repose encore en grande partie sur la liste type établie par F. Bordes. Les catégories d'outils sont limitées à quelques types morphologiques qui regroupent

3. François Bordes proposait le terme de «pointe Levallois » plutôt que "pointe levalloisienne », terme qui semblait les réserver au faciès « levalloisien » du Moustérien, c'est-à-dire au Moustérien de débitage Levallois, dans lequel une proportion importante d'éclats Levallois est conservée sans retouches (Bordes, 1961). des réalités hétérogènes. Cette catégorisation engendre une certaine déformation dans notre perception des volontés de l'artisan. L'outil typologique moustérien, selon ses dénominations traditionnelles, n'a qu'une faible consistance culturelle ou fonctionnelle. Nous atteignons ici une des limites de la méthode d'analyse.

La multitude des visages inférés à l'outil (longueur, largeur, type, module, matériau, traces d'utilisation, délinéation de tranchant, fonctionnalité...) découle du point de vue porté sur l'objet. Celui-ci présentera nécessairement autant de caractères propres que d'axes analytiques développés par le préhistorien.

L'outil et le rapport de l'artisan à l'outil relèvent avant tout du social. En tant qu'objet social, il ne présente pas nécessairement un lien strictement univoque entre ses potentialités et ses fonctions (Flichy, 1995). Ses usages résultent de propriétés inférées et du dialogue s'établissant entre l'objet et l'artisan. Un outil ne se résume donc ni à un processus de fabrication, ni à une forme, ni à une fonction, ni aux activités dans lesquelles il est engagé. Le produit fini, ici l'outil typologique, n'est pas un artefact comme un autre, c'est avant tout un objet, dans le plein sens de ce terme. Il faudrait plutôt, en Préhistoire, le considérer en tant que fraction d'objet, mais la nuance est, à ce niveau, sans conséquence majeure dans la mesure où l'outil typologique participe pleinement de cette fonction d'objet. Cette notion le différencie des déchets ayant servi à sa réalisation, attendu que la notion de déchets, sous-produits, produits d'intention première, ne présentent, à ce niveau, de réalité que pour l'observateur [le préhistorien], rendant plus périlleuse encore la compréhension des systèmes techniques fossiles. Cette nuance à l'esprit, un déchet pour le Préhistorique n'est pas un objet et ne possède pas le même statut.

Pour le «moustérianiste », l'analyse de l'outil constitue une tâche infiniment plus délicate qu'au sein des périodes postérieures de la Préhistoire, car les signifiants des outils du Moustérien, essentiellement des familles de racloirs, ne possèdent qu'une portée générique. Si une pointe de la Gravette, une pointe de Font-Yves ou une pointe de Châtelperron ont une résonance culturelle et fonctionnelle, le racloir, quand bien même nous l'appellerions moustérien, n'est qu'une catégorie anonyme indiquant la modification du tranchant d'un support par une série de retouches. Cette catégorie rend compte d'une réalité incertaine. Elle ne correspond pas même à une vaste famille regroupant un ensemble morphologiquement hétérogène, mais globalement comparable dans sa finalité. À vrai dire, le racloir présente une faible consistance analytique. 
Évidemment, ces considérations portent sur des tendances. Certaines classes d'outils du Paléolithique supérieur sont fortement hétérogènes et regroupent des réalités incertaines concernant lesquelles il est tout aussi délicat de statuer quant à la volonté de l'artisan. Toutes proportions gardées, et en ayant à l'esprit que ces remarques ont valeur d'orientation générale, les catégories d'outils au Paléolithique moyen agglomèrent des réalités disparates qui n'assument pas la même charge informative comparativement aux périodes récentes du Paléolithique.

L'ensemble des types établis par F. Bordes repose sur une description de la localisation et de la délinéation d'une modification de tranchant. Sa simplicité et son champ d'application, presque universel, expliquent largement le succès de la méthode. Ne serait-ce que d'un point de vue superficiel, morphologique, les types d'outils traditionnels du Moustérien représentent alors, de fait, des catégories fortement hétérogènes (fig. 9 à 12). Ce dispositif analytique apparaît cependant nécessaire et devrait, au premier degré d'une étude, être conservé. Cette nécessité est induite par une commodité de langage, mais surtout parce que le risque de voir se multiplier des catégories encore plus vides de sens est réel. Cette dernière éventualité est probablement plus fâcheuse que l'emploi d'un vocabulaire unique, quand bien même celui-ci serait réducteur ou stylisé. Si la préservation d'une nomenclature collective est une nécessité absolue, son utilisation doit, en revanche, s'exprimer en pleine conscience de ses limites.

Cette réflexion s'appuie sur deux tendances symétriques et propres aux industries du Moustérien: les assemblages présentent une grande homogénéité qui peut procurer une illusion de monotonie et jouissent, parallèlement, de caractères discrets individualisant fortement les complexes lithiques. La spécificité, car c'est bien là ce que nous recherchons, peut alors être abordée dans un second temps, afin d'évaluer les propriétés d'un corpus d'outils.

Ce second temps peut s'appuyer sur ces caractères discrets qui, bien qu'ils soient qualitatifs, voire stylistiques, illustrent les particularités des outillages et, par ricochet, notre perception des hommes et des sociétés du Paléolithique moyen.

Dans cet ordre d'idée, nous allons voir que certains racloirs transversaux peuvent être, rationnellement, des couteaux à dos abattu (!), et d'une manière plus générale que les pièces à dos sont plus fortement représentées qu'il n'y paraît. Leur reconnaissance nécessite de dépasser les perceptions premières, liées à l'utilisation des méthodes descriptives traditionnelles que nous venons d'aborder.

\section{APPLICATION, FORMES ET FONCTION : L'OUTIL DERRIÈRE LA GATÉGORIE}

Nous avons vu que les catégories typologiques du Moustérien présentent une faible pertinence quant à leur statut réel d'outil. Le racloir n'a pas pour principe de racler, quand bien même la tracéologie nous indiquerait une action de raclage. Ces familles d'objets dissimulent de nombreuses réalités, que l'on aborde la question selon ses attributs les plus empiriques - leurs fonctions effectives - ou - leurs propriétés structurelles -, ce que je regroupe ici sous la notion de rationalité.

L'outil moustérien apparaît alors intangible. Certains de ses aspects rationnels peuvent-ils être débusqués?

Il est possible de tenter un premier pas dans cette direction en tissant une trame analytique reposant sur des facteurs qualitatifs et stylistiques. Le processus analytique que je vais présenter s'appuie sur la riche industrie de Champ Grand (Slimak, 2004; Slimak dir., 2008). Il ressort de l'analyse du matériel retouché un faisceau d'indices permettant d'isoler certains principes de l'outil moustérien.

Les objets concernés sont des catégories de pièces convergentes (racloirs convergents et pointes moustériennes), ainsi que différents types de racloirs simples et doubles. Dans une moindre mesure, certains racloirs transversaux et racloirs partiels concourent de la même dynamique:

- l'analyse des racloirs convergents et des pointes moustériennes met en évidence, pour une majorité de ces catégories d'outils, une asymétrie marquée, induite par une incidence plus abrupte de la retouche sur l'un des bords de l'outil. Le tranchant opposé au bord le plus abrupt présente alors une retouche qui est relativement couvrante, parfois partielle ou irrégulière. Ces pièces possèdent au moins l'un de ces deux caractères, et souvent l'association des deux. Pour ces objets, ce traitement différentiel des tranchants est symptomatique. Ces objets sont souvent de délinéation droit-convexe, le bord droit de l'outil étant le plus souvent à la fois le plus convexe, le plus modifié et le plus abrupt;

- cette tendance des pièces convergentes trouve un parallèle direct au sein des racloirs doubles dont une partie présente des caractères comparables. Il y a traitement différentiel des deux bords et donc une volonté de l'artisan de réaliser une pièce dont les caractères de tranchant sont différents. Dans leur tendance, retouches différentielles et latéralité des outils sont similaires entre pièces convergentes et racloirs doubles;

- certains racloirs simples, convexes ou droits, dont l'incidence de la retouche n'est ni abrupte ni morpholo- 
giquement individualisable d'un racloir classique, sont opposés à un tranchant brut. Ce tranchant comporte systématiquement de fines ébréchures d'utilisation. Cette remarque est valable pour certains racloirs transversaux qui présentent des altérations d'utilisation sur le tranchant brut, naturellement déjeté et convergent depuis le talon du support vers la zone transversale retouchée. Parmi ces éléments, dont un seul bord est affecté par une transformation, quelques pièces non altérées portent des traces microscopiques et sont interprétables en tracéologie (Slimak dir., 2008). Sur ces éléments, le bord présentant des indications d'utilisation est systématiquement le tranchant brut.

Il apparaît ainsi que l'essentiel des pièces, racloirs simples, doubles, convergents, transversaux, pointes moustériennes, qui présentent les caractères susmentionnés, ont pour point commun de présenter une partie active opposée à un dos aménagé. Les caractères de ces dos ne correspondent pas aux caractères typologiques définissant un dos abattu - sensu Paléolithique supérieur - c'est-à-dire présentant une incidence abrupte à semi-abrupte et opposé à un tranchant naturel. La retouche aménageant une zone de préhension ou d'emmanchement, un dos stricto sensu, peut être similaire dans sa conception à une transformation de type racloir. C'est la cohérence générale des caractères de la pièce qui va permettre d'évaluer le statut de l'outil: zone active ou dos. Le rapprochement de ces différents outils - par ailleurs hétéroclites - ne concerne donc pas une réalité fonctionnelle mais porte sur l'un des caractères rationnels de l'objet. L'outil peut alors être abordé en tant que système, dont la cohérence est interne - propre à l'objet - et apparemment autonome de critères techniques récurrents - type de support, localisation et catégorie de retouche, forme et dimension...

Il est possible d'établir différentes dénominations à propos de ces dos. Les couteaux (ou pointes) à dos abattu résultant d'un aménagement abrupt à semi-abrupt opposé à un tranchant naturel, les couteaux (ou pointes) à dos retouché regroupant les pièces dont un des tranchants, modifié, correspond à un aménagement (souvent une altération) $\mathrm{du}$ tranchant naturel du support en vue de son maintien manuel ou emmanché. Pour ces éléments à dos retouché, le tranchant opposé peut être indifféremment naturel ou retouché.

Dans son application, une détermination aussi binaire est quasiment impossible en ce qui concerne les productions moustériennes qui ne se plient que rarement à des délimitations rigides. Cela induit que de nombreuses pièces sont morphologiquement ambiguës. L'incidence et l'aspect

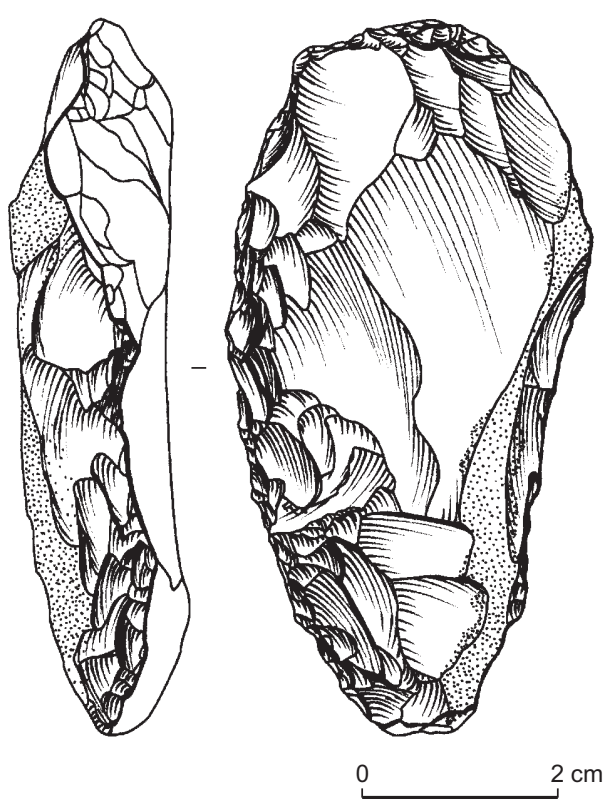

Fig. 9 - Racloir simple convexe en silex barrémo-bédoulien de Meysse (Ardèche) : grotte de Néron à Soyons (Ardèche), niveau II (dessin : L. Slimak, CNRS).

de la retouche, réalisés en vue de l'obtention de ces dos, sont variables. Cela n'affecte en rien leur caractère rationnel de pièces à dos. La dénomination de pièce (couteau, pointe) à dos façonné, appellation générique et regroupant pièces à dos abattu et pièces à dos retouché, permettrait d'éviter toute confusion entre ces deux réalités. Cette divergence est probablement anecdotique en ce qui concerne le Paléolithique moyen, dans la mesure où il est impossible d'établir une réelle typologie de ces outils qui serait fondée sur l'incidence de leurs aménagements.

Enfin, une dernière catégorie de pièces s'inscrit dans une même idée; ce sont des éléments dont un dos naturel est repris partiellement par une retouche prolongeant le caractère de dos naturellement présent sur le support. Le tranchant opposé pouvant, de manière indifférente, être brut ou retouché.

Il en ressort que la définition classique de la pièce à dos ne correspond qu'à une expression anecdotique comparativement à la représentation effective des pièces à dos dans certains ensembles du Moustérien. La conception et la perception de l'outil dos sont, à l'évidence, en décalage entre Paléolithique moyen et supérieur. Le caractère anecdotique de la pièce à dos dans l'équilibre typologique global du Paléolithique moyen serait alors, dans ce cas, le résultat d'un biais méthodologique: le fruit de notre perception de l'outil Moustérien. 


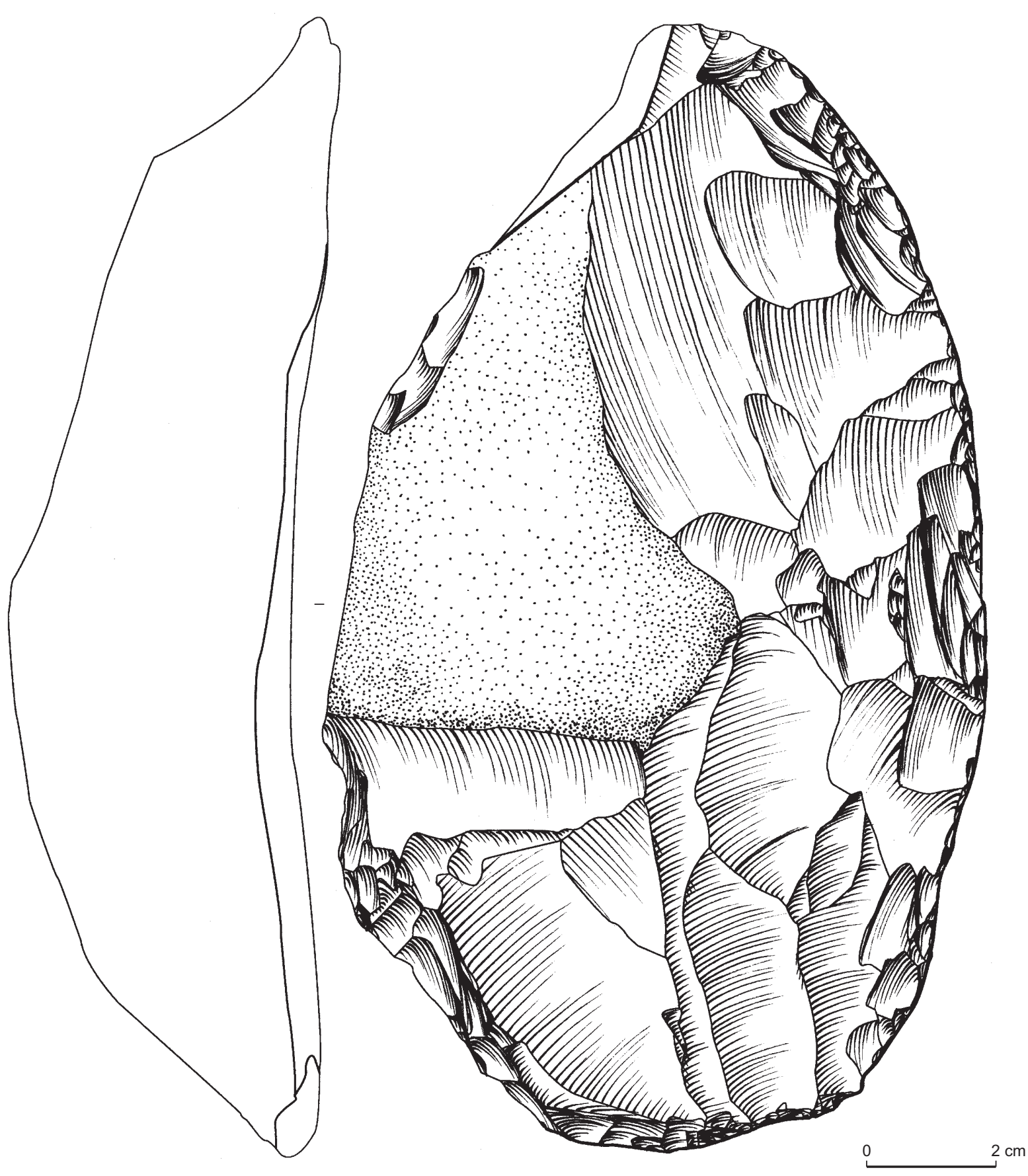

Fig. 10 - Racloir simple convexe en silex barrémo-bédoulien de Meysse (Ardèche) : grotte Mandrin à Malataverne (Drôme), niveau 6 (dessin: L. Slimak, CNRS).

Il est délicat de quantifier la proportion de ces pièces à dos, attendu que la démarche repose sur des caractères ténus. L'approche chiffrée est alors biaisée, elle exprime la quantification d'un attribut qualitatif. Cet attribut semble bien représenté dans certains ensembles du Moustérien.
À Champ Grand, celui-ci pourrait intéresser entre $30 \%$ et $50 \%$ des racloirs (Slimak, 2004).

La figure 13 illustre différentes catégories de racloirs interprétées en tant que pièces à dos. Ces outils montrent une asymétrie très marquée résultant de leur retouche 
et non des propriétés originales des supports. Le dos est exclusivement à droite et globalement convexe, hormis pour la pièce $n^{0} 6$ où celui-ci est rectiligne. Seule la pièce $n^{0} 1$ est une pièce à dos abattu stricto sensu-comprendre sensu Paléolithique supérieur -, présentant une retouche abrupte (ici croisée) opposée à un tranchant naturel. Sur les autres supports, la retouche affectant le bord opposé au dos peut être irrégulière (pièces $\mathrm{n}^{\mathrm{os}} 4$ et 5 ), marginale (pièces $\mathrm{n}^{\mathrm{os}} 2$ et 3), voire écailleuse et caractéristique d'un façonnage de type racloir (pièces $n^{\text {os }} 6$ et 7 ). En dépassant leur diversité morphologique, technologique et typologique, on s'aperçoit que ces pièces révèlent un principe unique: leur attribut de dos prime sur leur hétérogénéité morphotechnique. Enfin, dans cet exemple, non seulement le dos est dextre, mais l'incidence et la convexité (sauf pour la $\mathrm{n}^{\circ} 6$, délinéation droite) maximales de leur dos sont localisées dans le tiers distal de l'outil et font ainsi apparaître une pointe plus ou moins acuminée.

Moins évident au premier abord, tant l'œil est habitué à l'exercice typologique classique, certains racloirs transversaux sont de véritables pointes à dos retouché. L'axe technique de l'éclat dissimule le caractère rationnel de l'objet (fig. 14).

Si la multiplication des couteaux à dos dans certains ensembles du Paléolithique moyen permet de tisser une trame superficielle entre ces complexes et certaines expressions du Paléolithique supérieur, le rapport à l'outil est finalement si différent que le rapprochement sous un abord - rationnel, fonctionnel - a pour corollaire un éloignement sous un autre - comportemental. Conceptuellement, nous perdons d'un côté ce que nous gagnons de l'autre.

Sous cet angle, le saut dialectique du Paléolithique moyen au supérieur n'est ni typologique ni fonctionnel, mais repose sur le rapport de l'artisan à l'outil et à ses productions.

\section{ORIENTATIONS}

Ces réflexions ne sont que des ouvertures, un point de vue heuristique. Elles visent à déstabiliser un caractère monolithique de certains des principes d'analyse du Paléolithique moyen en pointant un certain nombre de failles dans notre acception des systèmes techniques du Moustérien. Dans cette approche, aucune des démarches traditionnelles, typologiques - sensu Bordes - ou technologiques - sensu Boëda - n'est remise en cause. En revanche, leurs attributions et leurs champs d'application sont remodelés.

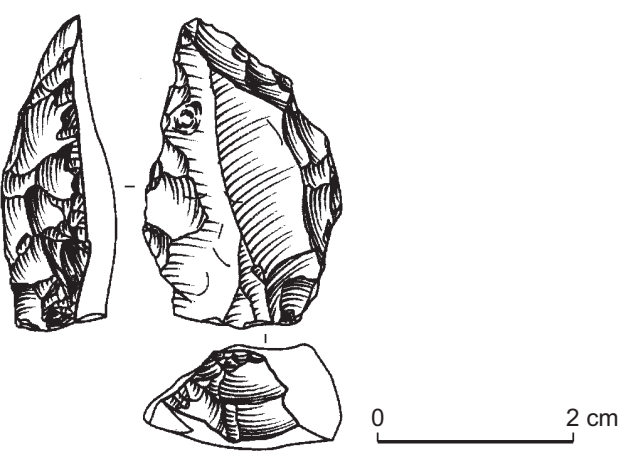

Fig. 11 - Pointe moustérienne en silex barrémo-bédoulien de Meysse (Ardèche) : grotte de Néron à Soyons (Ardèche), niveau II (dessin : L. Slimak, CNRS).

La finalité d'une telle démarche est de dégager un espace conceptuel au sein duquel une plus grande souplesse analytique et une plus grande clarté dans la délimitation de nos concepts permettraient de rendre compte, de manière opérante, de la diversité des systèmes techniques du Moustérien.

Aucun parallèle univoque n'est établi entre les notions de rythme - continu/discontinu - et les concepts Discoïdes ou Levallois. En tant qu'outil analytique, ces notions sont affranchies de toute connotation chronologique ou culturelle. Ces notions permettent d'analyser l'espace conceptuel propre aux débitages Levallois et donc d'en définir leurs limites. Cette analyse induit un recadrage des débitages Levallois sur leurs principes conceptuels originaux, plutôt que d'en élargir l'entendement, par glissements sémantiques successifs. Cette déviation progressive de la sphère du Levallois s'exprime en dehors de tout cadre analytique et ne permet ni une délimitation ni une structuration rationnelle du domaine d'attribution propre au concept. La détermination des marges d'un concept est un processus influant sur sa définition propre, tout autant que sur les possibilités qui nous sont offertes de discerner et de définir ce qui n'en relève pas. Une telle démarche autorise non seulement l'émergence de concepts originaux, mais permet d'évaluer, pour chacun d'entre eux, leurs attributions, leurs champs d'application et leurs degrés de parenté.

Venons-en à l'outil. Un ensemble moustérien ne livre jamais deux outils identiques. Il apparaît alors délicat de dégager des propriétés récurrentes permettant de rendre compte des relations s'établissant entre l'artisan et ses objectifs. Au premier abord l'outil moustérien semble répondre d'une géométrie incertaine. Ce trait apparaît avec force dans sa propension à ne pas se résumer au travers de 

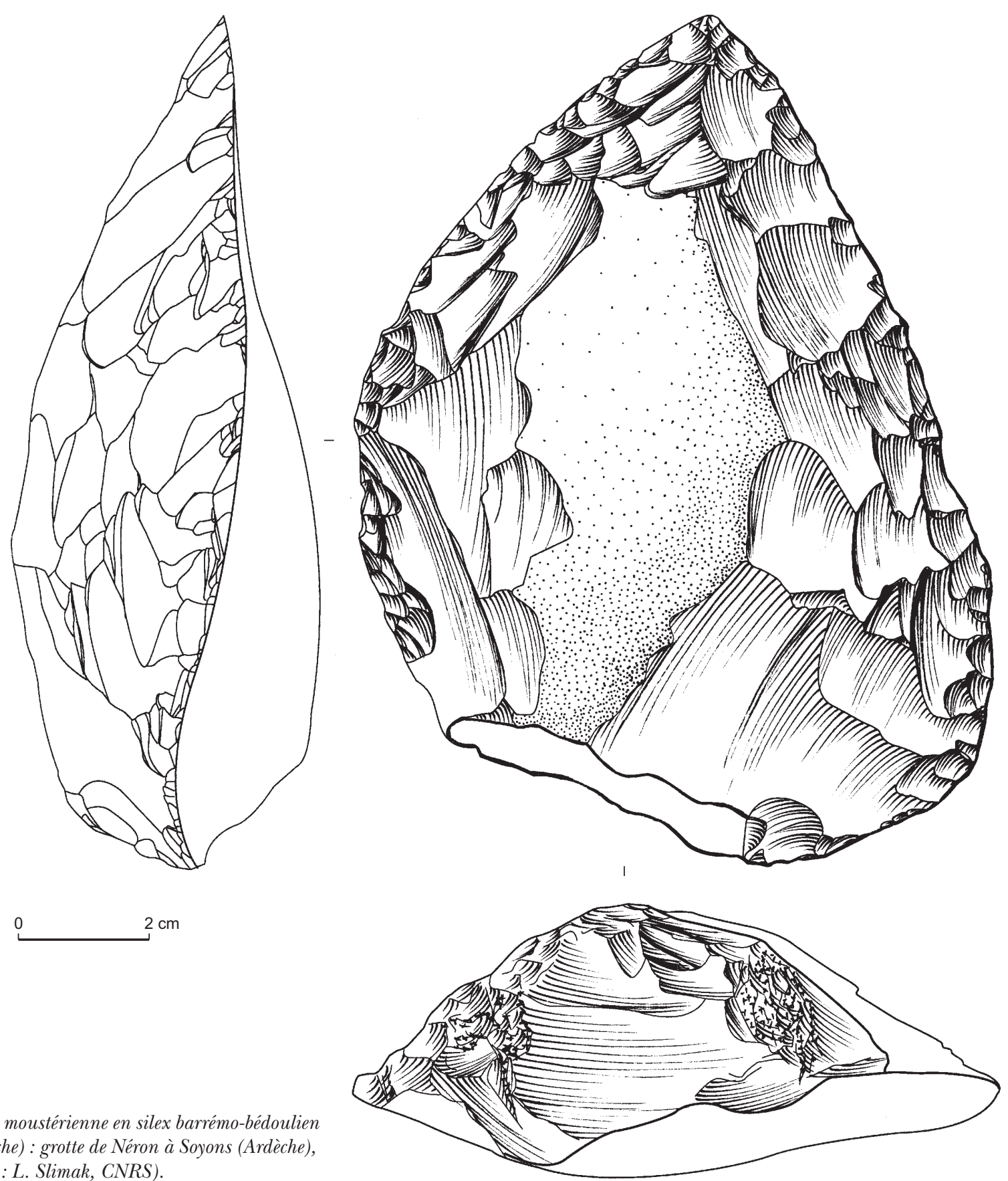

Fig. 12 - Pointe moustérienne en silex barrémo-bédoulien de Meysse (Ardèche) : grotte de Néron à Soyons (Ardèche), niveau I (dessin : L. Slimak, CNRS).

racloir serait le fruit d'une évolution graduelle de l'outil au cours de son existence (Dibble, 1987).

Bien au contraire, si le racloir témoigne d'une impressionnante diversité morphologique, sa structure et son équilibre, en tant que système, paraissent particulièrement stables. Nous abordons peut-être à ce niveau l'un des principes 

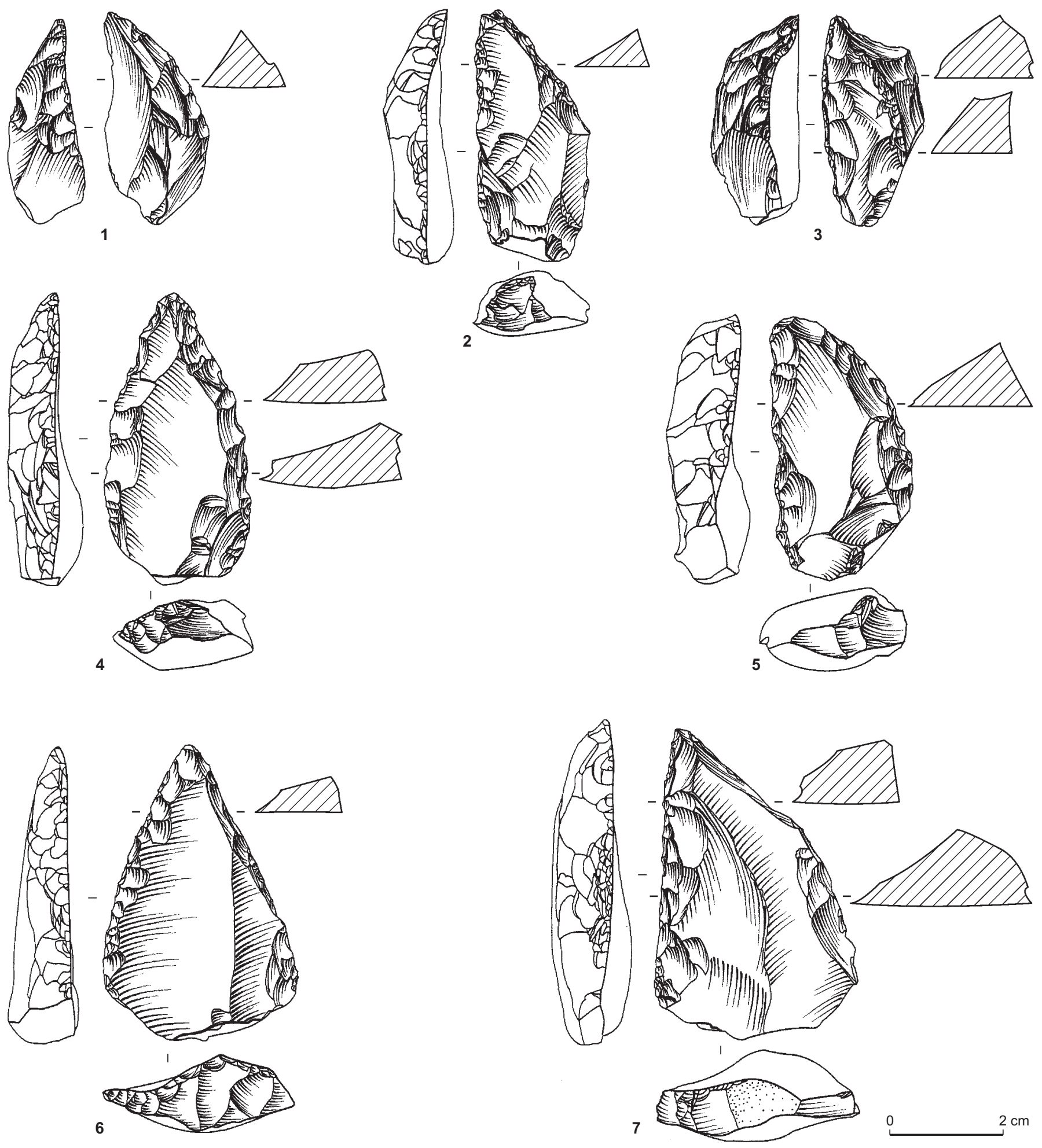

Fig. 13 - Différentes catégories de racloirs typologiques présentant un caractère rationnel de pointes à dos retouché découvertes à Champ Grand à Saint-Jean-Saint-Maurice-sur-Loire (Loire) (dessin : L. Slimak, CNRS). 


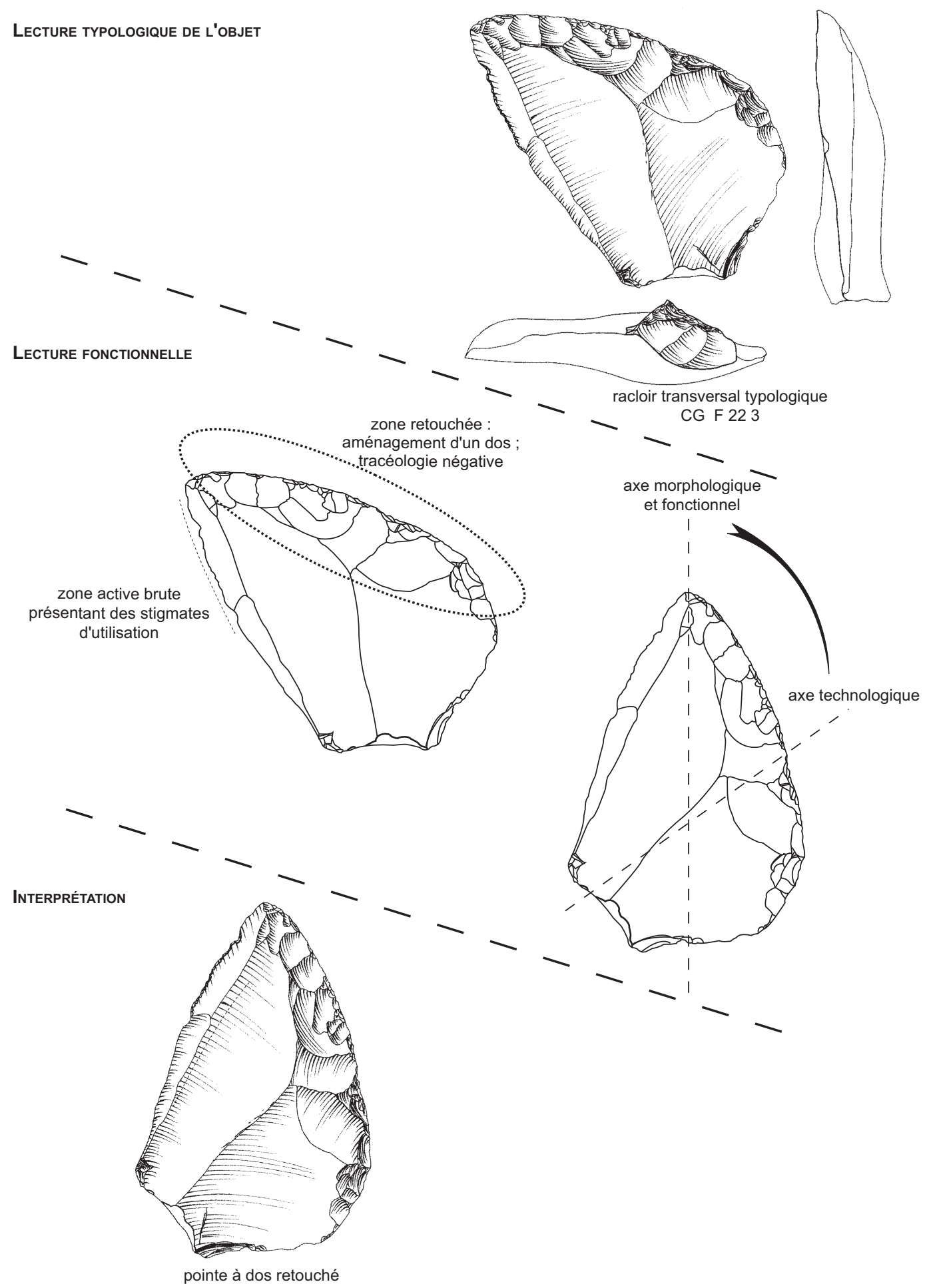

Fig. 14 - Racloir transversal selon la typologie de F. Bordes, rationnellement pointe à dos retouché découverte à Champ Grand à Saint-Jean-Saint-Maurice-sur-Loire (Loire) (dessin : L. Slimak, CNRS). 
fondamentaux de l'outil moustérien qui semble fonctionner en tant que système. Chaque outil correspond alors à une unité indépendante répondant à sa logique propre. Cette propriété confère aux industries du Paléolithique moyen un caractère modulaire. L'outil exprime sa structure sur des principes internes, indépendants des attributs techniques les plus immédiatement perceptibles (forme, typologie, origine technique du support). Cet aspect presque autarcique de chaque élément du système s'exprime donc dans la structure de l'objet; chaque outil d'un ensemble moustérien est une création unique, mais aussi dans les relations reliant l'outil à l'ensemble du système technique, auquel il participe pourtant: il n'est possible de relever aucun lien équivoque entre une catégorie technique et une catégorie typologique, par exemple.

Reste à définir si ces propriétés, de l'outil et des systèmes techniques, correspondent à un état particulier de l'organisation de ces sociétés - pouvant correspondre à un palier évolutif - ou relèvent, à proprement parler, d'un domaine comportemental - donc de portée génétique. Ces propriétés nous permettraient alors de distinguer non pas le Paléolithique moyen du Paléolithique supérieur, mais certaines conceptions et logiques qui seraient, quant à elles, propres aux sociétés néandertaliennes.

\section{BIBLIOGRAPHIE}

\section{BOËDA E.}

1988 : « Le concept laminaire : rupture et filiation avec le concept Levallois ", in OTTE M. (DIR.), L’Homme de Néandertal, Actes du colloque international de Liège - 8La Mutation, Liège, Université de Liège (coll. ERAUL, 35), p. 41-59.

1990 : « De la surface au volume : analyse des conceptions des débitages Levallois et laminaires ", in FARIZY C. (DIR.), Paléolithique moyen récent et Paléolithique supérieur ancien en Europe, Nemours, APRAIF (coll. Mémoires du musée de Préhistoire d'Île-de-France, 3), p. 63-68.

1993 : « Le débitage discoïde et le débitage Levallois récurrent centripète ", Bulletin de la Société préhistorique française, 90, p. 392-404.

1994 : Le Concept Levallois : variabilité des méthodes, Paris, CNRS Éditions (coll. Monographie du Centre de recherches archéologiques), $283 \mathrm{p}$

\section{BORDES F.}

1961 : Typologie du Paléolithique ancien et moyen, Bordeaux, Delmas (coll. Publications de l'Institut de Préhistoire de l'Université de Bordeaux, mém., 1), 2 vol., 85 p. et 108 pl.

\section{DIBBLE H. L.}

1987 : « The Interpretation of Middle Paleolithic Scraper Morphology ", American Antiquity, 52-1, p. 109-117.

\section{FLICHY P.}

1995 : L'Innovation technique, récents développements en sciences sociales : vers une nouvelle théorie de l'innovation, Paris, La Découverte (coll. Sciences et sociétés), 255 p.

\section{PELEGRIN J.}

1986: Technologie lithique: une méthode appliquée à l'étude de deux séries du Périgordien ancien (Roc de Combe, couche 8 - La Côte, niveau III), Paris, Université Paris-X-Nanterre, thèse de Doctorat, $584 \mathrm{p}$.

1995 : Technologie lithique : le Châtelperronien de Roc de Combe (Lot) et de La Côte (Dordogne), Paris, CNRS Éditions (coll. Cahiers du Quaternaire, 20), 298 p.

\section{SLIMAK L.}

2003 : «Les débitages Discoïdes moustériens : évaluation d'un concept technologique ", in PERESAni M. (DIR.), Discoid Lithic Technology : Advances and Implications, Oxford, British Archaeological Reports (coll. BAR International Series, 1120), p. 33-65.

2004 : Les Dernières expressions du Moustérien entre Loire et Rhône, thèse de Doctorat, Université de Provence, 865 p.

2007 : « Le Néronien et la structure historique du basculement du Paléolithique moyen au Paléolithique supérieur en France méditerranéenne ", Comptes rendus Palevol, 6, p. 301-309.

\section{SLIMAK L. (DIR.)}

2008 : Artisanats et territoires des chasseurs moustériens de Champ Grand, Aix-enProvence, Maison méditerranéenne des sciences de l'homme (coll. Artisanats et Territoires, 1), $432 \mathrm{p}$.

\section{SLIMAK L., LUCAS G.}

2005 : « Le débitage lamellaire, une invention aurignacienne ? ", in LE BRUNRICALENS F., BORDES J.-G., BON F. (COORD.), Productions lamellaires attribuées à l'Aurignacien : chaînes opératoires et perspectives technoculturelles, Actes du XIV congrès de l'Union internationale des sciences préhistoriques et protohistoriques, Luxembourg, Musée national d'Histoire et d'Art (coll. ArchéoLogiques, 1), p. 75-100. 